$\begin{array}{lll}\text { Vol. XV. } & \text { OCTOBER, } 1934 & \text { No. } 58\end{array}$

\title{
Orígínal Dapers
}

\section{OBSERVATIONS ON RECORDS OF LOGAL EPILEPTIC CONVULSIONS *}

\author{
$\mathrm{By}$ \\ D. DENNY-BROWN AND E. GRAEME ROBERTSON, LoNdon
}

INTRODUCTION

Method

Protocols

Discussion .

The Cerebrospinal Fluid Pressure

The Effect of Sleep

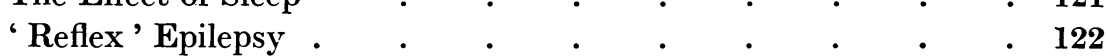

The Incoordination of the Epileptic Discharge . . . . . 124

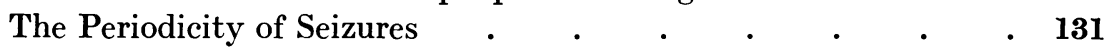

Conclusions

\section{INTRODUGTION}

THE varied phenomena of epilepsy and the many clinical problems with which it is associated present a perennial source of interest to the clinician. The convulsion itself presents many problems which become the more interesting the more closely they are observed, though the rapid sequence and concurrence of a number of manifestations tend to confuse clinical observation. Some of the events lend themselves to analysis by graphic record, and the following account concerns records of cerebrospinal fluid pressure, respiration, and muscular movement in six patients. Though such records fall far short of any exact quantitative measurement of muscular contraction, careful control by clinical observation allows them a measure of accuracy, and in their èssential feature, namely sequence of events in time, they appear to us to be reliable evidence.

* From the National Hospital for Nervous Diseases, Queen Square, London. 


\section{METHOD}

The pressure of the cerebrospinal fluid was recorded by means of a mirror tambour capsule which reflected a beam of light to a moving strip of bromide paper. The pressure was transmitted from a wide lumbar puncture needle to the glass capsule by means of thick rubber tubing of small inside diameter. A small sterile chamber intervened between the needle and the tubing, and here the cerebrospinal fluid reached a level by compressing the air within the capsule system. The capsules used are identical with those we ${ }^{5}$ have described in detail for use in the measurement of vesical pressure, and are calibrated after each experiment by measuring the deflection produced by water columns of various heights. The periodicity of the system is well damped by the slight fluid movement ; the latency is negligible at the rates of recording used. The patient lay on the least active side, on a wide couch, for periods up to three hours. Movements have been recorded by the attachment of pressure balloons over the chest wall, and over the muscle concerned, with due precaution to avoid the possibility of movement by other muscles. Changes in pressure within the balloons was transferred to capsules with mirrors which recorded on the same bromide paper. The record of movement, though sufficiently accurate for time and general amplitude, cannot be used for accurate quantitative analysis. The latency of movement was tested separately and found to vary from 0.004 sec. with short tubing (head, trunk and upper limb) to $0.01 \mathrm{sec}$. with longer tubing (lower limb). In records as rapid as those in figs. 25-29 there was no measurable difference between air transmission and direct beam from muscle. Time was recorded by a rotary Rayleigh wheel driven by an electrically operated tuning fork. When action currents have been recorded a Matthews oscillograph system has been used, with leads from the muscle by needle electrodes.

\section{PROTOGOLS}

Case I. Regular series of predominantly left-sided convulsions at brief intervals. Previous history of sensory and inhibitory attacks. No loss of consciousness. Pain in affected limbs. Spastic left-sided weakness due to right parietal astrocytoma.

M.B., age 54, years, female, was admitted under the care of Dr. Gordon Holmes in November, 1932. There was no pertinent family history or previous illness. In September, 1922, there occurred the first of a long series of brief attacks at intervals from several days to several weeks. In each of these there occurred a sensation of something rushing over her head, lasting several seconds, during which she could neither speak nor move. There was no loss of consciousness, and she would be observed only to stand still with fixed expression. At times the patient would turn round during an attack, and on some occasions the left arm and leg would become somewhat stiff, at times only the left leg. In other attacks there was a pricking sensation of the left side of the neck and tongue. The frequency of these attacks was much reduced by luminal, though they continued at long intervals for the next three years.

In one night in May, 1925, there occurred four generalized convulsions with loss of consciousness and preceded for a short interval by a severe pain in the left leg 'as 
though someone were trying to pull it out of its socket.' She was not incontinent nor did she bite her tongue. She was admitted to another hospital and a right parietal decompression performed, after which the left arm and leg were completely paralysed but recovered in four weeks. Attacks of varied pattern continued. For a brief interval she had warnings of the 'wave passing over her head,' but from that time onwards she had no aura. There were attacks of tonic and then clonic spasm in the left arm and leg, attacks in which she was unable to use the left arm for a few seconds, and also attacks in which the left leg would feel quite numb for perhaps a half-minute. In none was there loss of consciousness, and the attacks recurred at intervals of a few hours to a few weeks, the intervals being longest when she was taking luminal.

On November 15, 1932, there developed a numbness of the left arm and leg, which lasted continuously for five days, and the patient then commenced to have very frequent attacks of spasm of flexion, lasting 15 seconds to one minute, at the left hip and knee, accompanied by severe pain in the left thigh. As the attacks continued progressive loss of voluntary power in the left lower limb occurred. There was no involvement of the right side. At the onset of later attacks the left arm was drawn up, but she was still able to move the fingers.

Attacks observed on November 22, 1932, presented the following features: If the patient were talking speech ceased suddenly and her face stiffened. There was a variable degree of stiffening of both arms and the right leg; the left lower limb became tonically flexed at the hip and knee with fine jerking movements at the ankle. After some 10 to 12 seconds the jerking increased in amplitude and clonic movements at the hip and knee commenced and lasted about 15 seconds. In some attacks the left arm adducted strongly. The patient was able to move the right arm to order during the attacks and stated that she was fully conscious and suffering pain in the dorsum of the left foot and in the left thigh.

There was slight bilateral papillœdema and slight weakness of voluntary movement of the left side of the face. All movements in the left upper limb, especially those at shoulder and elbow, were weak, and there was spasticity in the adductors of the shoulder and flexors of wrist and elbow. There was no power of voluntary movement in the left lower limb, the extensors of which were extremely spastic. The tendon reflexes of the left limbs were exaggerated and the left plantar response was extensor. There was no defect of sensation. The decompression was under no increase in tension. The cerebrospinal fluid showed no abnormality.

She was treated by luminal gr. 2 three times daily. On November 20 she had 60 attacks, the following day 30, and on the day of examination 50 attacks. Her temperature was irregular, rising from $97 \cdot 4^{\circ}$ to $99 \cdot 6^{\circ} \mathrm{F}$.

Subsequently, on November 25, the previous decompression opening was explored by the late Sir Percy Sargent and the cerebral cortex of the parietal lobe was found to be replaced by gelatinous tissue which extended to the median longitudinal fissure. A mass about 2 inches in diameter was removed and histological examination proved it to be an astrocytoma. She made a good recovery from operation and was discharged from hospital with a spastic weakness of the left arm and leg.

Analysis of attacks recorded on November 22, 1932.-In this patient besides a manometer to record the pressure of the cerebrospinal fluid through the lumbar puncture needle it was arranged that the movements of the upper abdomen and of the left great toe were recorded, together with the fluctuations in pressure at the decompression opening on the right side of the head. We had the kind assistance of Dr. Gilbert Hall in recording the attacks. Seven fits were recorded in their entirety, and the characteristic records shown 


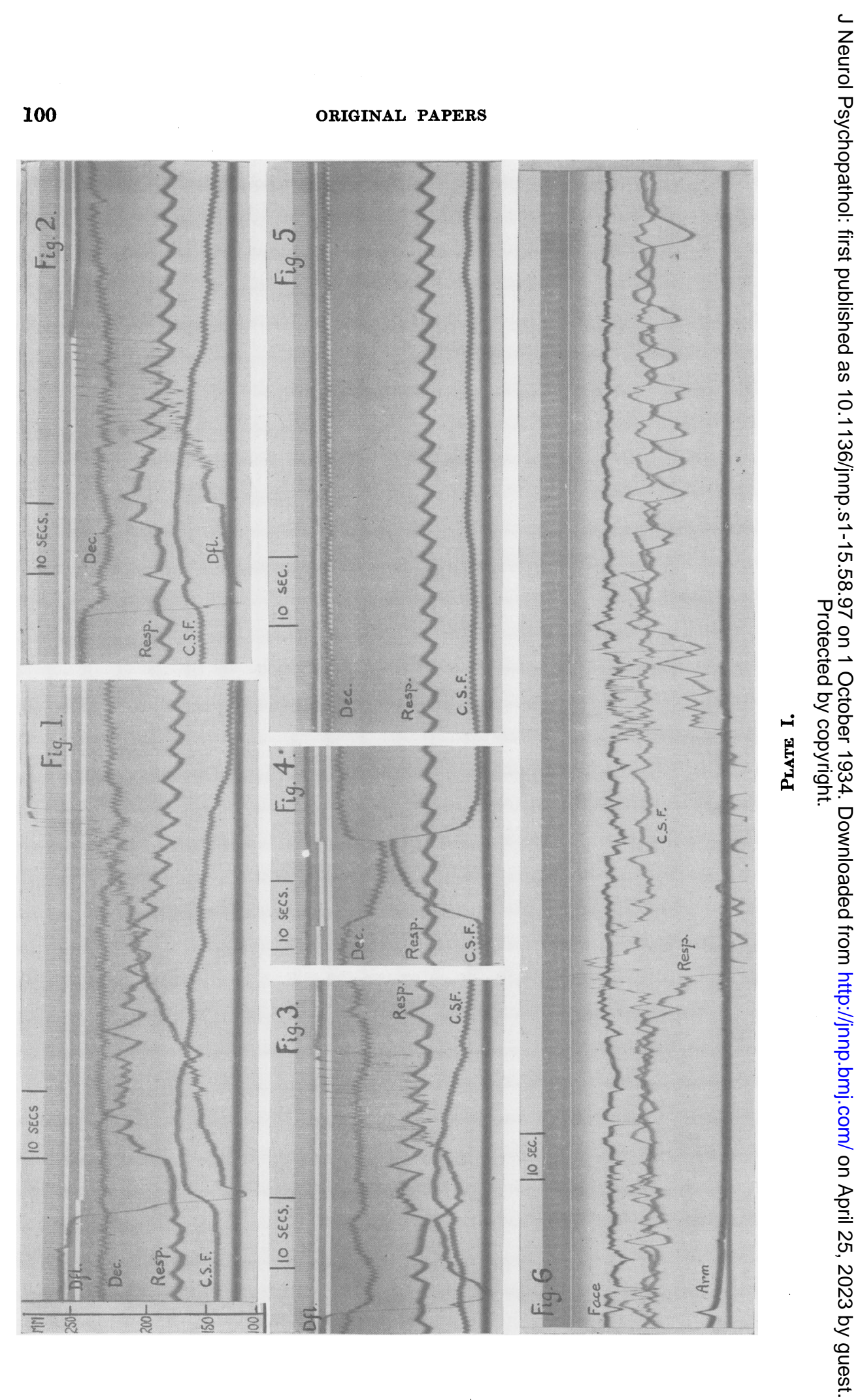


in figs. 1, 2 and 3 show the course of the attacks. As will be seen in all these figures the onset of the attack was an abrupt dorsiflexion of the great toe, which was in all instances succeeded by a momentary cessation of respiration shown by the abdominal recorder. The cerebrospinal fluid pressure commenced to rise, usually as early as four-fifths of a second after the onset of dorsiflexion of the great toe (figs. 1 and 2), although in isolated instances, such

\section{DESGRIPTION OF PLATE I}

Plates I-VII. From records of epileptic attacks made photographically by movements of beams of light on a moving strip of bromide paper. All records read from left to right. Time is recorded electrically in fine vertical strokes at the top of the record at one-fifth second intervals. As the closeness of the vertical strokes in slow records tends to make them illegible after reduction, intervals of 10 seconds have been superimposed for the convenience of the reader. In Plates VI and VII the speed of the record renders this unnecessary. The tracing of the manometer recording cerebrospinal fluid pressure is marked C.S.F. in all records. In figs. $8,11,13,14$ and 15 the cerebrospinal fluid pressure is recorded both by delicate and coarse manometers the tracings of which are marked C.S.F. 1 and C.S.F. 2 respectively. The scale of tension at the commencement of each record refers to the calibration of the cerebrospinal fluid pressure (in $\mathrm{mm}$. of water) as registered by the delicate manometer. The tracing which records chest movement is marked Resp. (or in some cases where abdominal movement was recorded, Abd.). The deflection is downwards for inspiration in all cases. In each case a record is made of some muscular movement prominent in the attack and the lettering of these records is described in the legend of each figure.

Plate 1. Fig. 1.-Record of epileptic attack in Case I, 12 minutes after the last preceding attack. The uppermost moving line Dfl. registers movements of the left great toe and makes downward excursion for dorsiflexion and small upward excursion for plantar flexion. The tracing Dec. records the pressure within a balloon bound over the decompression opening in the skull, and shows an excursion with each pulse beat, and was also affected by movements of the face and neck. The pressure within the balloon at the commencement of the record was $12 \mathrm{~cm}$. of water. The small movement of the foot three seconds before the commencement of a fit was probably voluntary.

Fig. 2.-Tracings and C.S.F. tension scale as in fig. 1, here showing a fit of shorter duration, 10 minutes after that recorded in fig. 1 and two and a half minutes after an intervening fit. The higher initial cerebrospinal fluid pressure was consequent upon some apnœa occurring after the hyperventilation of the intervening fit.

Fig. 3.-Tracing and C.S.F. tension scale as in fig. 1. Two minutes 40 seconds after the attack featured in fig. 2, showing delayed rise in cerebrospinal fluid pressure.

Fig. 4.-Tracings and C.S.F. tension scale as in fig. 1. To show the effect of compression of the left jugular vein in the interval signalled by the duration of the fall in the upper white line.

Fig. 5. - Tracings and C.S.F. tension scale as in fig. 1. To show irregular fluctuations in the cerebrospinal fluid pressure during a period of drowsiness some 10 minutes after the attack recorded in fig. 3.

Fig. 6.-Showing myoclonic twitching followed by major attack in Case II. The uppermost darker tracing (Face) shows a downward deflection for contraction of the left corner of the mouth. The figure commences with the occurrence of the first movement after a long period of quietude. Following the period shown in this figure the twitching disappeared after a few seconds and hyperpnœa occurred before the patient again became quiet. In the centre of the figure contraction of the trunk muscles in the tonic stage of the convulsion causes prolonged displacement of the chest recorder (Resp.). C.S.F. pressure as in scale in fig. 7 . 
as fig. 3, the rise was much longer delayed. This rise of pressure amounted at times to as much as $65 \mathrm{~mm}$. of cerebrospinal fluid, as in fig. 1. After a first rapid rise it usually maintained an almost steady plateau, commencing to subside only when abrupt irregularity in the toe-recorder showed the first evidence of the clonic stage. Whether regular beats of clonus continued from this time onwards, as in fig. 2, or became established only very late in the attack, as in fig. 1, the reestablishment of original cerebrospinal fluid pressure was gradual and progressive. As will be seen in all three records the later fall of fluid pressure is related more directly to the reestablishment of regular respiration, as shown by the abdominal recorder. Where the abdominal movement at the onset of the attack is slight, as in fig. 3, the cerebrospinal fluid pressure may rise rapidly, late in the tonic stage, when the abdominal and thoracic muscles eventually participate in the attack.

The attempted record of the pressure at the decompression opening was found to be partly independent of the cerebrospinal fluid pressure change, as is seen in the figures shown, and inspection at the time revealed that, however applied, the fixation of the balloon at the decompression orifice was affected by the powerful movement of the face and neck musculature, which in most attacks commenced to occur within from three-fifths to one second after the first movement of the great toe. The absence of any real independence in cerebrospinal fluid and intracranial pressure is shown by light jugular compression when particular care was exercised not to disturb the fixation of the balloon over the decompression (fig. 4).

It will be noted that the resting cerebrospinal fluid pressure varied from 135 to $150 \mathrm{~mm}$. of fluid owing to the continual fluctuations which were observed during the intervals between fits. These were particularly marked when the patient was drowsy, as in fig. 5 , but in this patient were not observed to be greater than $20 \mathrm{~mm}$. in range. They were not accompanied by any parallel change in respiration or abdominal posture. In one attack the pressure following the fit remained higher $(110 \mathrm{~mm}$.) than it was before the attack (100 mm.). In the other attacks, as in figs. 1, 2 and 3, the pressure was lower for an interval of some 15 to 30 seconds following the attack. There was no change preceding the attack in any of the records.

Case II. Generalized convulsions from early age. Three periods of status epilepticus at long intervals. Epileptic myoclonus, with ' reflex' provocation. Major attacks with predominantly left-sided convulsion. Repeated development and recovery of postepileptic hemiplegia.

G.T., age 12 years, female, admitted under the care of Dr. Gordon Holmes, in July, 1931, May, 1932, and January, 1933. No family history of epilepsy. Generalized convulsions commenced at the age of four years, and continued at the rate of two to four a week. The attacks were almost always nocturnal, usually with biting of the tongue and rarely with incontinence. Luminal administered regularly controlled the attacks, but the parents refused to continue its use. In July, 1931, generalized 
convulsions, with loss of consciousness, incontinence and biting of the tongue, commenced to occur at hourly intervals. There was sufficient recovery in the intervals to talk, but after two weeks the left upper and lower limbs were noticed to be weak between the attacks. A week later, when she was admitted to hospital, the attacks were still occurring at the rate of about one an hour, each followed by a drowsy stage which lightened only for a brief interval before the onset of the next attack. The left limbs were weak, particularly in the grasp of the hand and in dorsiflexion of the foot, and both were slightly hypertonic, with increased tendon jerks. The abdominal reflexes were absent on the left side and the left plantar response was extensor.

In the attacks the head was turned to the left and the eyes upwards and to the right, the left arm and leg showed clonic movements, the right arm and leg rigidity. In some attacks twitching of the face preceded that of the left arm and leg. The evening temperature was usually elevated-on the thirteenth day, when the fits were lessening, reaching $102^{\circ} \mathrm{F}$. Only once was the morning temperature high $\left(99^{\circ} \mathrm{F}\right.$.). There was no correspondence between the number of attacks and the height of the temperature. She was treated by luminal and paraldehyde (fig. 30, status I). The intervals between attacks lengthened and the left hemiparesis gradually disappeared.

On discharge from hospital the patient remained free from attacks for one month and at the end of that period, at intervals of seven to 14 days, there commenced a series of momentary nocturnal attacks of twitching of the eyelids with generalized stiffness and trembling. Nocturnal enuresis was frequent. At the beginning of May, 1932, the frequency increased to two to three attacks every night and within a few days attacks became very frequent indeed, and generalized convulsions occurred. The smaller attacks now occurred in the daytime. In these, with the trembling there was a little cry, the lips would become blue, and she was unable to talk for a moment or two. The large attacks took the form of turning of the head to the right with flexion of the arms at the elbows, hands clenched, eyes closed, with some cyanosis of the lips. The head then turned to the left and the left arm extended. The attack subsided with some clonic jerking of the left arm and was followed by drowsiness. The total duration was some 30 seconds. In other attacks the head turned to the left with eyes to the right, the right upper limb was held extended, the left flexed. In the intervals between the attacks the left arm and leg were hypotonic, but there was at this time no defect in motor power, except for a left facial weakness. The tendon reflexes were increased in the leftupper limb, but equal in the lower limbs. Abdominal reflexes were absent and plantar responses both flexor. Blood pressure 120/70.

Neither paraldehyde nor morphia controlled the attacks, which were temporarily reduced in number, but not controlled by luminal gr. 5 intravenously. The attacks diminished in severity but the total frequency was little altered. A left-sided hemiparesis developed, with marked left-sided hypotonia, and extensor plantar response. There was defective sensation of position and passive movement with astereognosis in the left hand. At this time records of the fits were made. The temperature rose only on two occasions, once in the evening to $99^{\circ}$ and once in the morning to $99 \cdot 8^{\circ} \mathrm{F}$. (fourteenth and sixteenth days-fig. 30, status II). Treatment by bromides, chloral and luminal (up to gr. 4 a day by mouth) was continued and the number of attacks lessened and eventually ceased. Ventriculography showed no displacement or deformity of the ventricles.

Attacks continued at very rare intervals during the next six months, when again, in spite of continuous treatment by luminal, tonic convulsions of the left arm and leg commenced to become very frequent. On this occasion a left-sided hemiparesis, with flaccid arm and slight increase of tone in the lower limb, developed. There was loss of two-point discrimination and astereognosis in the left upper limb. The attacks were as before, except that both head and eyes were turned to the left. Respiration was profoundly affected. The left plantar response was observed on several occasions 
to be extensor for a period of three minutes after an attack, the right remaining flexor throughout. The attacks gradually lessened in frequency and finally ceased (fig. 30, status III). The temperature showed only occasional elevation to $99 \cdot 2^{\circ} \mathrm{F}$. on the morning of the third day and to $99^{\circ} \mathrm{F}$. on the evening of the twenty-sixth day. When the attacks were most numerous the temperature varied between $97^{\circ}$ and $98 \cdot 4^{\circ} \mathrm{F}$.

On March 7, 1933, an exploration of the cerebral cortex by a right fronto-temporoparietal osteoplastic flap was made by Mr. Julian Taylor. The subarachnoid lakes appeared to be unusually distended. There was no evidence of tumour. The patient made a rapid recovery, and has remained free from fits.

Analysis of attacks recorded on June 10 and 15, 1932.-The facial movements in this patient showed continued irregular brief twitchings occurring singly or in groups. At times twitching of the left arm and of the left side of the trunk also occurred. In records of these twitchings there appeared to be no direct relationship between the occasional myoclonic twitches in the face and those which were observed in the trunk and wrist as shown in figs. 6 and 8. At long intervals, usually when all twitching had gradually subsided in the preceding 10-20 minutes, abrupt and violent twitching would break out in the left side of the face, trunk and left arm concurrently. This would partly subside sometimes after five or 10 seconds, leaving for an interval more frequent twitching in the face. The cerebrospinal fluid pressure at these times showed only some small fluctuations corresponding to those of the chest. At other times, as in fig. 6, the initial abrupt movements in the face and trunk gave way to a more maintained tonic posture lasting up to a minute, during which time some isolated jerky movements of the face, arm and trunk occurred. For a variable period following this a rapid irregular clonic twitching of the face remained. With the initial outburst in such cases the cerebrospinal fluid pressure showed irregular jerks of increase, but fluctuations occurred corresponding in time to the larger trunk movements. In the intervening periods when the patient was drowsy, when myoclonic twitching was rarely observed or at times absent for periods of minutes, it was noticed that a sudden stimulus caused awakening accompanied by a rapid sequence of myoclonic twitches. In this way a myoclonic attack or even a tonic-clonic convulsion appeared to be elicited by stimulation. Such a convulsion is recorded in fig. 7. Here, a loud noise having proved ineffective, sudden cold was applied to the left calf. Immediately there resulted movement of the left arm and left leg and after two seconds rapid twitches of wide amplitude appeared in the left side of the face and the left limbs. These ceased after a further two seconds, when there was a powerful contraction of the left side of the trunk with cessation of respiration. After a cessation for some four seconds this tonic contraction was renewed in more powerful form and a tonic convulsion was established. The clonic stage which ensued passed into irregular myoclonic twitching lasting many minutes. The cerebrospinal fluid pressure undergoes small fluctuations which reflect only the more abrupt movements of the chest. 


\section{Case III. Two isolated outbursts of status epilepticus, with long intervening} interval. Compulsion aura. Predominantly right-sided attacks with speech defect. Postepileptic paralysis. Asthmatic subject.

L. K., male, age 31 years, admitted to the National Hospital October, 1933, under the care of Dr. Hinds Howell. From his youth he had suffered from attacks of asthma, with occasional eruptions of giant urticaria. In October, 1932, without warning he had a generalized convulsion, with loss of consciousness, succeeded by stupor, which was interrupted 24 hours later by another convulsion. During the next nine days, of which he has no memory, he remained in a stuporose state. He states that in the subsequent few days he felt 'completely exhausted' and remembers having six to eight attacks in which his right arm jumped and the elbow flexed involuntarily. He then remained free of attacks until April, 1933, when he commenced to experience momentary ' sensations' in which he felt that he ' must do something but did not know what.' Two weeks later one of these sensations was followed by a generalized convulsion with loss of consciousness. Treatment with luminal was then commenced and he had no further attacks until a generalized convulsion followed two weeks after the discontinuing of such medication in June, 1933. He was admitted to hospital five days later and no abnormal physical signs were found. The cerebrospinal fluid was normal. Skin tests showed a moderate reaction to the meat group, in particular to beef. On discharge he remained well except for periodical asthmatic attacks, which were relieved by ephedrin. At the height of one such attack the occurrence of one of his 'sensations' was accompanied by subsidence of the asthmatic attack. On September 26, 1933, after momentary confusion of mind he lost consciousness for a period of two hours, after which he remembers twitching all over. His asthmatic trouble, previously occurring nightly, was lessened for 48 hours. Four days later the patient had an attack in which he felt impelled to say something but was unable to speak, following which his right arm was raised. Similar attacks followed, and in the intervals, which became progressively shorter, he felt exhausted and had difficulty in expressing himself. In this state, two days later, on October 2, when the attacks were recurring every 20 minutes, he was admitted to hospital. The unpleasant sensation of necessity for speech, with the production of an unrecognisable sound which trailed off into ' a silence,' continued to precede each attack. He was able to understand what was said to him during an attack but could make no response of any kind. Observed attacks commenced with an irregular groaning, twitching of the upper lip, turning of the head to the right, and similar deviation of the eyes with occasional reassertions of forward gaze. His right arm became flexed at elbow and wrist. After two minutes the periodic groaning ceased, he would smile, and after a momentary clonic twitching of both corners of his mouth, he would turn his head forward and speak rationally. A series of such attacks was recorded.

Upon examination slight weakness of the lower right side of the face and of the right side of the palate and tongue were apparent. Motor power, tone and limb reflexes were normal, except that the right plantar response was less flexor than the left, and immediately after an attack was extensor for a few minutes. The cerebrospinal fluid contained 20 cells per c.mm., total protein 0.030 per cent.; globulin tests were negative and the Lange normal. Ventricular fluid contained 19 cells per c.mm. and protein 0.03 per cent. Ventriculography failed to reveal any abnormality of the cerebral ventricles.

The day after his admission some attacks involved the face alone, and while the right side was drawn up in spasm the left eye would close intermittently and the mouth would be drawn first to one side and then to the other. In some attacks the right arm was involved in clonic convulsion, in others in tonic flexion with tonic extension of the 

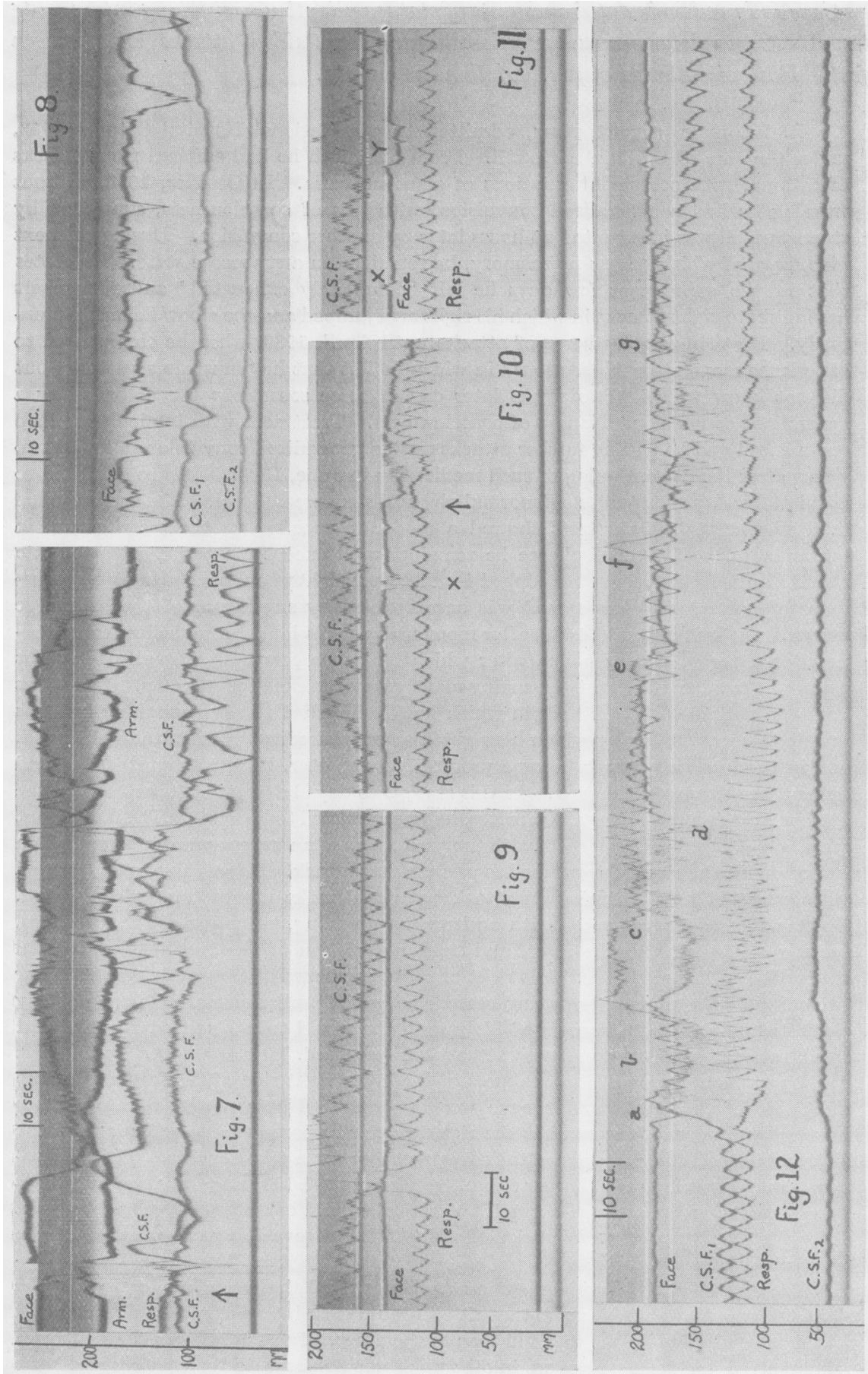
right leg. After some attacks he was completely aphasic for 10 minutes. Examination now showed increased weakness of the right side of the face, palate, tongue and right hand. The right arm was hypotonic and both lower limbs showed a slight increase in extensor tone. The tendon reflexes were equal and brisk in the lower limbs, with patellar clonus; the plantar responses were extensor during the attacks but plantar flexion replaced this reaction in the intervals. On the day of admission he had 33 attacks and three drams of paraldehyde were administered per rectum. The following day he had 26 attacks and was treated by two hypodermic injections of morphia (gr. $\frac{1}{6}$ and gr. $\frac{1}{4}$ ), 4 drams of paraldehyde per rectum, and luminal gr. $1 \frac{1}{2}$ three times daily. The last was alone continued thereafter. On October 4 he had nine attacks, on October 5, five, and then was free from attacks during the rest of his stay in hospital. There was no elevation of temperature with the attacks.

Analysis of attacks recorded on October 2, 1933.-In this patient the use of a wide-bore lumbar puncture needle and a sensitive manometer enabled us to obtain a more than usually clear record of the pulse and respiratory waves. The close correspondence between the movements of the chest and the cerebrospinal fluid pressure is evident in fig. 9, where for a brief interval the patient is instructed to cough. Even speech, to the extent of the enunciation of one word, also causes a brief rise of pressure (fig. 11). The initial fall, and after a brief interval the sudden rise in cerebrospinal fluid pressure, are distinctly seen, also the subsequent slight fall of pressure for a period of some 30 seconds due to the increased movement of the chest with increased ventila-

\section{DESGRIPTION OF PLATE II}

Plate II. Fig. 7 (Case II). - As in fig. 6, with the addition of a tracing (Arm) recording occurrence of flexion of the left wrist as an upward excursion. The patient having previously been asleep, cold is applied to the left calf at the moment indicated by the arrow directed upwards. A powerful myoclonic jerk is followed after a few seconds by the commencement of an attack in which tonic movement of the face with expiration is followed by a long period of myoclonus.

Fig. 8.-Tension scale for C.S.F. 1 as in fig. 7. The upper record shows by downward deflections a series of myoclonic contractions of the left side of the face. Respiration is not recorded.

Fig. 9 (Case III).- To show the effect of a cough in the duration of fall of the upper white line.

Fig. 10 (Case III).-Time, C.S.F. pressure, and respiration as in fig. 9. To show the earliest change in C.S.F. pressure as recorded by an extremely sensitive manometer. A balloon over the right corner of the mouth causes a manometer tracing (Face) to move downwards when contraction occurs. At the point marked $\mathbf{X}$ the patient made some small voluntary facial movement while at the point marked $(\uparrow)$ a convulsion commences.

Fig. 11.-C.S.F. tension, etc., as figs. 9 and 10. To show effect of two brief periods of speech marked $\mathrm{X}$ and $\mathrm{Y}$.

Fig. 12.-Complete record of a fit in Case III. Time, C.S.F. tension and other records as in figs. 9, 10 and 11, with the addition of a tracing of a coarse manometer C.S.F. 2. Some momentary displacements of the upper white line are made by an electrical signal by the observer to indicate at $a$ slight turning of the head towards the affected side, at $b$ the first groaning sound, and at $c$ more powerful turning of the head to the affected side ; at $d$ the patient nodded his head and said 'I'm all right.' From $e$ to $g$ the patient talked and at $f$ coughed to order. 
tion immediately following the apnœe. Jugular compression was followed by a rapid rise of cerebrospinal fluid pressure within two seconds. This manometer showed well the earliest changes during a fit (fig. 10), and a fully recorded fit with both coarse and delicate manometers is shown in fig. 12 . The pressure is seen to commence to rise apparently as the first event in the attack, although close inspection of the record reveals that the accompanying respiration is more brief than its predecessors. The tonic stage of the attack lasts only five seconds in the face, and the steep rise of cerebrospinal fluid pressure during this interval thereafter ceases, falling very slightly during subsequent clonus and rising sharply again 15 seconds later where the facial movements again become tonic for a brief interval. The period of apnca here outlasts the initial rise of pressure and at the end of the attack the pressure falls only very slowly, being delayed for some 20 seconds by a renewed outburst of clonic twitching of the face. The original level was reached only after four minutes from the termination of the attack. The recorded signals during this fit indicate that vocalisation occurred with or slightly after the first movement of the face and that the secondary rise of cerebrospinal fluid pressure begins seven seconds after the commencement of turning of the head. It will be noted that the regular rapid movements of the chest show no change at this time. All these features were confirmed in the record of another fit.

Case IV. Onset of epilepsy three months after the evacuation of a frontal abscess. Three periods of status epilepticus. Left-sided attacks, with postepileptic paralysis and sensory disturbance. Coincidence with reflex stimulation.

L. S., age 27 years, female, admitted under the care of Dr. James Collier in March, 1930, January, 1931, and November, 1933. In 1922 the patient had a septic appendix removed. In 1925 there began severe frontal headaches with failing vision. In January, 1926, she was admitted to St. George's Hospital where a right frontal cerebral abscess was drained. In March, 1926, she commenced to suffer from attacks of unconsciousness preceded by twitching of the left arm and leg, occurring at long intervals for four years, but gradually increasing in frequency. Two days before her admission to hospital on March 14, 1930, attacks involving the left arm and sometimes the left side of the face commenced to occur every few minutes. For one to two minutes after the cessation of such attacks the left arm was useless. The attacks were partly controlled by the administration of four-hourly doses of paraldehyde and ceased five days after admission. She had only one rise of temperature, to $99 \cdot 8^{\circ} \mathrm{F}$., during this time. The attacks then ceased completely, but bromide and luminal were continued. In January, 1931, attacks of shaking of the left arm lasting a few seconds and occurring every half-hour commenced. Three days later the attacks spread further; commencing with flexion of the left elbow and wrist and turning of the head to the left, the left side of the face and trunk were affected later in the attack. After the onset of the fit there was no movement of the head, which could be turned in the opposite direction to order. There was no deviation of the eyes. Consciousness was not lost. Again weakness of the left side of the face and the left arm, with diminished tone and slight weakness of the left arm, was observed in the intervals between the 
attacks. There was complete astereognosis, loss of two-point discrimination and diminution of postural sensibility in the left hand.

The attacks were treated by paraldehyde and rapidly became fewer, and thereafter under luminal treatment she remained free from attacks until October, 1933, having had a pregnancy in this interval. A single nocturnal fit then occurred. A week later, after a day of infrequent attacks, the fits recurred every three to five minutes. Paraldehyde treatment controlled the attacks to some extent until the fifth day after their onset, when they commenced to occur every two to five minutes. The attacks continued during waking and sleeping hours for 14 days in spite of intravenous administration of luminal gr. 3 on three occasions and 2 drams of paraldehyde by rectum fourhourly. During this period there gradually developed a complete flaccid paralysis of the left side of the face, arm and leg. In the three weeks following cessation of the attacks the paralysis slowly improved, leaving slight weakness of the left side of the face, astereognosis in the left hand and loss of two-point discrimination in the left hand and left foot. The temperature reached $99^{\circ} \mathrm{F}$. on one occasion only, after the cessation of the attacks.

At the time records were made of the attacks the sequence of events was as follows : For a second or two the patient appeared to be aware that an attack was imminent. Her head turned slightly to the left and she complained of a tingling sensation in the left hand. The left arm was abducted at the shoulder and partially pronated at the elbow, with extended wrist. The wrist then flexed and the fingers extended. The left leg was then abducted at the thigh and feet were inverted and extended. The right leg showed a similar movement of the foot only. The left side of the face was slightly contracted. When these attitudes had been maintained for 10 to 15 seconds clonic movements appeared in the left side of the face, in the left arm, abdominal muscles and slightly in the lower limb. Clonic movements were sometimes seen in the deep muscles of the right side of the neck. These movements appeared to occur in the same order as the tonic spasm and persisted longest, sometimes in the left hand, sometimes at the left corner of the mouth. The patient was conscious and able to speak during the attacks.

In the intervals between the attacks the left arm and leg were in a state of complete flaccid paralysis. There was no demonstrable weakness of movements of the upper face, but the left side of the lower face remained completely immobile upon attempted voluntary movement. For associated movements the whole left side of the face moved well but not so strongly as the right. On emotional expression the left side of the lower face moved earlier and more completely than the right. The palate was slightly weak on the left side and the tongue deviated to the left. There was no weakness of sternomastoids or trapezil. There was slight weakness of the abdominal muscles on the left side. The tendon jerks were increased in both arms, and both knee and ankle jerks were present and equal. The abdominal reflexes were absent on the left and present on the right. The plantar reflex was flexor on the right, absent on the left. In the upper and lower limbs there was complete loss of position sense, appreciation of passive movement, stereognosis and two-point discrimination, with loss of position sense and two-point discrimination in the left lower limb. The blood pressure was $170 / 110 \mathrm{~mm}$. Hg. both during and between the attacks. The cerebrospinal fluid was reported to contain no cells, protein 0.050 per cent., Nonne-Apelt negative, Pandy positive. Lange and Wassermann reactions negative. The blood calcium was $10 \mathrm{mgm}$. per cent. in one period of attacks.

Analysis of attacks recorded on November 7, 1933.--In this patient attacks were occurring at brief intervals of surprising regularity. In consecutive attacks which were recorded the intervals from onset to onset were as follows : 


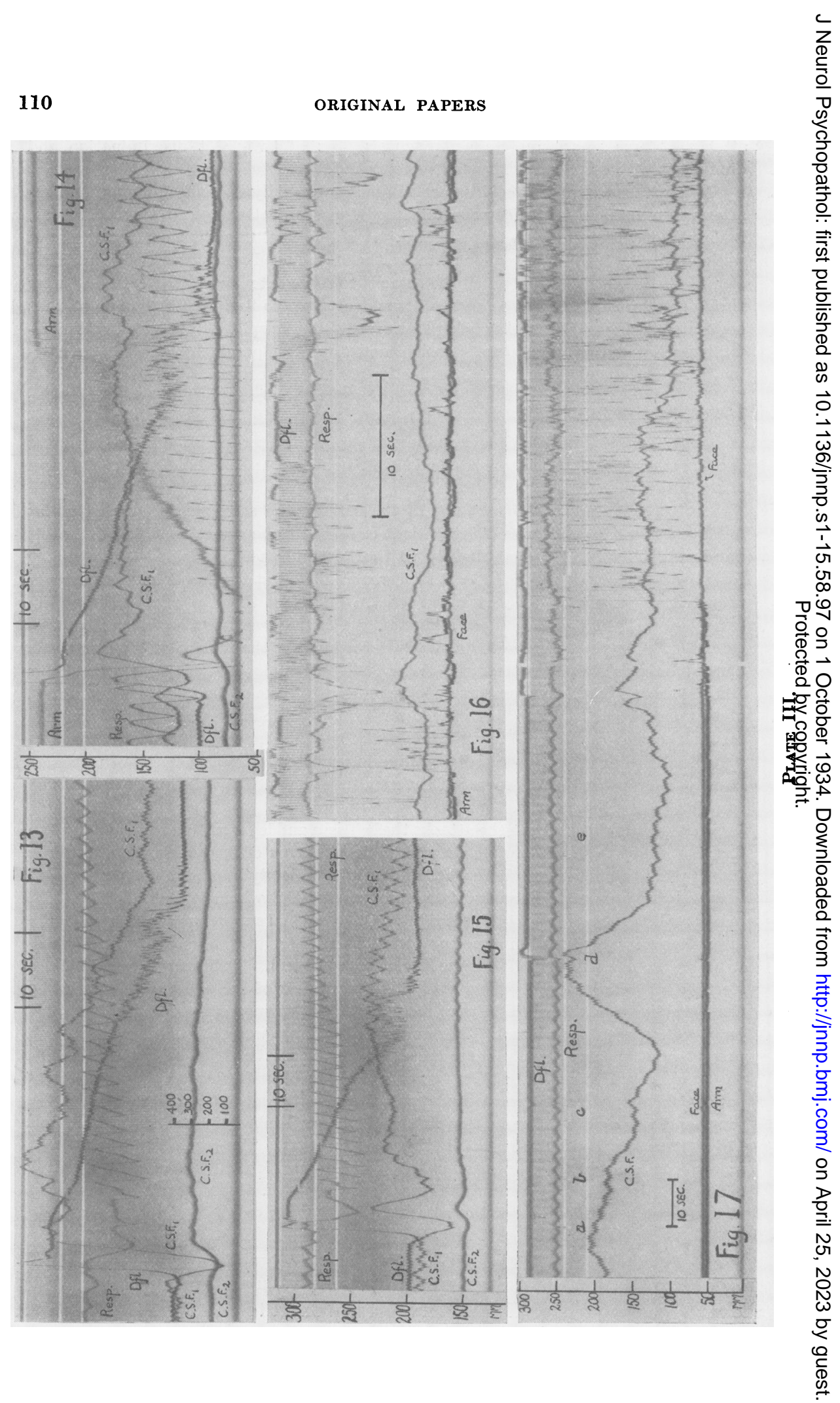


$3 \mathrm{~min}$., $3 \mathrm{~min} .8 \mathrm{sec}$, $3 \mathrm{~min} .8 \mathrm{sec}$, $3 \mathrm{~min} .30 \mathrm{sec}$., $3 \mathrm{~min} .54 \mathrm{sec}$., $3 \mathrm{~min} .36 \mathrm{sec}$, $3 \mathrm{~min} .36 \mathrm{sec}$; after two attacks in which the interval was not recorded, a further consecutive series showed the following intervals: $4 \mathrm{~min}$. 3 sec., 3 min. 22 sec., 3 min. 25 sec., 3 min. 37 sec., 3 min. 32 sec., 3 min. 28 sec., $3 \mathrm{~min}$. 12 sec., $3 \mathrm{~min}$. $28 \mathrm{sec}$., $3 \mathrm{~min}$. $48 \mathrm{sec}$., $3 \mathrm{~min}$. $32 \mathrm{sec}$. The slight variation was not accounted for by variation in the duration of each attack, which showed independent slight fluctuation.

In these attacks, examples of which are shown in figs. 13, 14 and 15, there was constantly an initial fall in cerebrospinal fluid pressure followed by a rapid and high rise, which thereafter subsided gradually during the clonic stage of the attack. The initial fall in pressure was usually, but not always, preceded by a slight plantar flexion of the foot. It was regularly preceded by a somewhat deeper inspiration than before, and the succeeding sharp rise was contemporaneous with a very deep expiration, which heralded the onset of the tonic stage of chest movement. This relationship is clearly shown in fig. 14. In all attacks the tonic chest movement subsided more rapidly than the tonic stage in the limbs. Where the movement of the arm was recorded, as in fig. 14, it was found that the clonic stage ceased earlier in this member than in the lower limb. Particular care was taken to protect the decompression opening in the skull from any adventitious pressure. The cerebrospinal fluid pressure returned to its previous level only some 30 to 60 seconds after the termination of the attack. It will be noticed that the delay in the final

\section{DESGRIPTION OF PLATE III}

Plate 11I. Fig. 13 (Case IV).- The scale of tension recorded by C.S.F. 1 is shown at the commencement of fig. 15. The dark tracing Dfl. moves upward with dorsiflexion of the left foot.

Fig. 14.-As fig. 13, with the addition of a manometer (Arm) which by its downward deflection shows the occurrence of clasping of the left hand. Though by observation the attack appeared to commence at the same moment in foot, hand and face, this record reveals some delay in the movement of the hand.

Fig. 15 (Case IV).-As fig. 13. To show a more brief attack, with less initial rise of cerebrospinal fluid pressure.

Fig. 16 (Case V).-Tension of cerebrospinal fluid as in fig. 17. To show by more rapid recording the relationship between the individual clonic movement in face, arm, thorax and foot. Time intervals in one-fifth second are seen as vertical white strokes at the top of the record. The uppermost tracing Dfl. shows downward movement for dorsiflexion of the left foot. Below it (Resp.) movements of the thorax are recorded with downward displacement for inspiration. Below the C.S.F. pressure tracing (C.S.F.) two superimposed lines show movements of the face and arm. The darker (Arm) shows clasping of the hand; the lighter (Face), which makes only small deflections, records contraction of the left corner of the mouth.

Fig. 17 (Case V).-Shows the commencement of a generalized myoclonic convulsion following stimulation of the patient during sleep. The figure begins with high C.S.F. pressure during sleep. At the signal recorded in the upper white line at $a$ the dorsal aspect of the left forearm was pricked once, at $b$ once, at $c 10$ times in succession, again at $d$, and 12 times at $e$. 
fall of pressure is often related to a similar delay in the return of the chest wall to the depth of full expiration.

The effect of various stimuli which appeared to be provocative of attacks was recorded. Friction of the left hand and stimulation of the sole of the left foot were tried on many occasions, on most of which they were without effect, and when they were succeeded by an attack the time of the attack was within the limits of natural variation of the intervals above noted.

\section{Case V. Congenital syphilis. Rapid development of dementia with} involuntary twitching, the latter improved by treatment. Recurrence six months later with right-sided epileptic seizures and rapid onset of right hemiplegia. Subsequent onset of left-sided myoclonic convulsions.

W. S., age 19 years, male, admitted under the care of Dr. Hinds Howell in February, 1934. He was born at full time of apparently healthy parents who had previously had three healthy children and one miscarriage. At the age of five years it was discovered that the right eye was blind and the vision of the left eye impaired. When eight years old he attended a school for the blind and made satisfactory progress. At the beginning of 1933 he was noticed to make purposeless movements of his tongue, and he had difficulty in speaking, especially when excited. In May, 1933, unsteadiness in walking and purposeless movements of his hands and legs were noticed. In July, 1933, he was admitted to hospital. His speech was almost unintelligible owing to dysarthria and he showed an advanced degree of mental impairment. In the right eye there was no. perception of light : the visual acuity of the left eye was $6 / 36$ corrected. The right eye was small with a band of corneal degeneration, probably congenital. The left eye showed some synechiæ from old iritis and a widespread syphilitic choroidoretinitis. There was constant nystagmus of moderate rate and equal components, both at rest and on lateral deviation of the eyes. The left pupil reacted to direct light. Convergence was poor. The tendon reflexes were exaggerated and he showed irregular choreiform movements of the limbs and constant grimacing. The cerebrospinal fluid showed 42 cells, total protein 0.060 per cent., globulin positive, Lange 4544332100 . Wassermann reaction full positive in cerebrospinal fluid and blood. After a course of intravenous therapy speech was a little clearer and the involuntary movements were less pronounced.

In November, 1933, a left hemiplegia occurred suddenly, with complete recovery during the ensuing night. He then remained well until the end of January, 1934, when for a period of 24 hours he developed extreme restlessness of both upper limbs and trembling of the lower limbs. Three days later he again had difficulty in moving his legs and after two further days, on February 4, at frequent intervals, for 24 hours, the right arm and leg commenced a series of spasms each lasting about 10 minutes, with the gradual development of a complete right-sided hemiplegia followed by cessation of twitching. At that time, on the morning of admission, the left corner of the mouth and the left hand commenced to twitch and there was occasional vesical incontinence. He was admitted to hospital in a stuporous condition. Attacks of twitching were observed in the left corner of the mouth and left eyelids; more rarely there was twitching of the jaw. The left hand was rarely quiescent, displaying purposive movements, and in addition irregular myoclonic movements of the fingers, or of one finger alone, and occasionally of the wrist, elbow and shoulder. All movements were much increased by interference, which promoted a directed resistance, and ceased during periods of quietude. Occasionally the left thigh flexed or abducted and the 
great toe dorsiflexed. The left upper and lower limbs were of normal tone and no defect of muscular power was demonstrable. Reflex grasping could be elicited by stimulation of the palm of the left hand. The right side was completely paralysed and immobile, with increased flexor tone in the upper limb and extensor spasticity in the lower limb. The tendon reflexes were present and equal in the upper limbs, brisk and equal in the lower limbs, the abdominal reflexes were absent on the right side, and both plantar responses were extensor. Cooperation for sensory examination was not possible, but pin-prick evoked more general reaction from the left side than from the right. The cerebrospinal fluid was reported to contain 23 cells per c.mm., total protein 0.037 per cent., globulin weakly positive, Lange 1223442210. Wassermann reaction full positive in cerebrospinal fluid and blood.

On February 6 four drams of paraldehyde per rectum reduced the involuntary movements to occasional clonic twitches of the left side of the face and the left hand, which still occurred in greater number, together with resistive movements of the left arm when the patient was disturbed. His temperature varied between $100 \cdot 2^{\circ}$ and $103^{\circ} \mathrm{F}$., his pulse between 108 and 138. Minor attacks continued in spite of three further administrations of four drams of paraldehyde, until his death two days after admission. A postmortem examination disclosed considerable dilation of all cerebral ventricles due to granular ependymitis, with chronic arachnoiditis, and accompanied by considerable cortical degeneration (dementia paralytica).

Analysis of attacks recorded on February 5, 1934.-In this patient the cerebrospinal fluid pressure was found to be undergoing wide fluctuations during the intervals of sleep or stupor between the attacks (figs. 18-21). There was no accompanying fluctuation of respiration. The maximum pressure was $300 \mathrm{~mm}$., with falls to $160 \mathrm{~mm}$., lasting 40 seconds, as at $a$ in fig. 18, with considerable fluctuation in extent and duration. Before the onset of an attack the average pressure would fall gradually and slowly to about $150 \mathrm{~mm}$. Rapid irregular movements of the face, of the foot and of the chest would commence concurrently and increase in irregularity, the pressure at first showing only fine rapid fluctuations corresponding to the movements of the chest. After an interval of some 30 to 40 seconds the movements of the chest would become more violent and the pressure rise slightly. About this time rapid irregular clonic movement commonly commenced in the upper limb. The subsidence of the attack usually began with subsidence of the facial movements, but isolated irregular twitching of face, arm, trunk and leg continued to occur for many seconds, with sometimes an irregular fresh outburst in the arm or trunk, as in figs. 19 and 20. With the subsidence of twitching the pressure at first fell to the region of $100 \mathrm{~mm}$. of water for an interval and rose in a series of increasing fluctuations to reach its original high level. The patient was at this time noted to be returning to his original sleeping state from which the attack had awakened him.

Rapidly moving records of the twitching during an attack showed the complete independence of the myoclonic twitching in the face, arm, trunk and leg (fig. 16). The series of events recorded in figs. 18-21 was repeated in two other complete records of attacks, differing only in the relative violence and duration of movement. Occasionally a few myoclonic twitches of the arm 


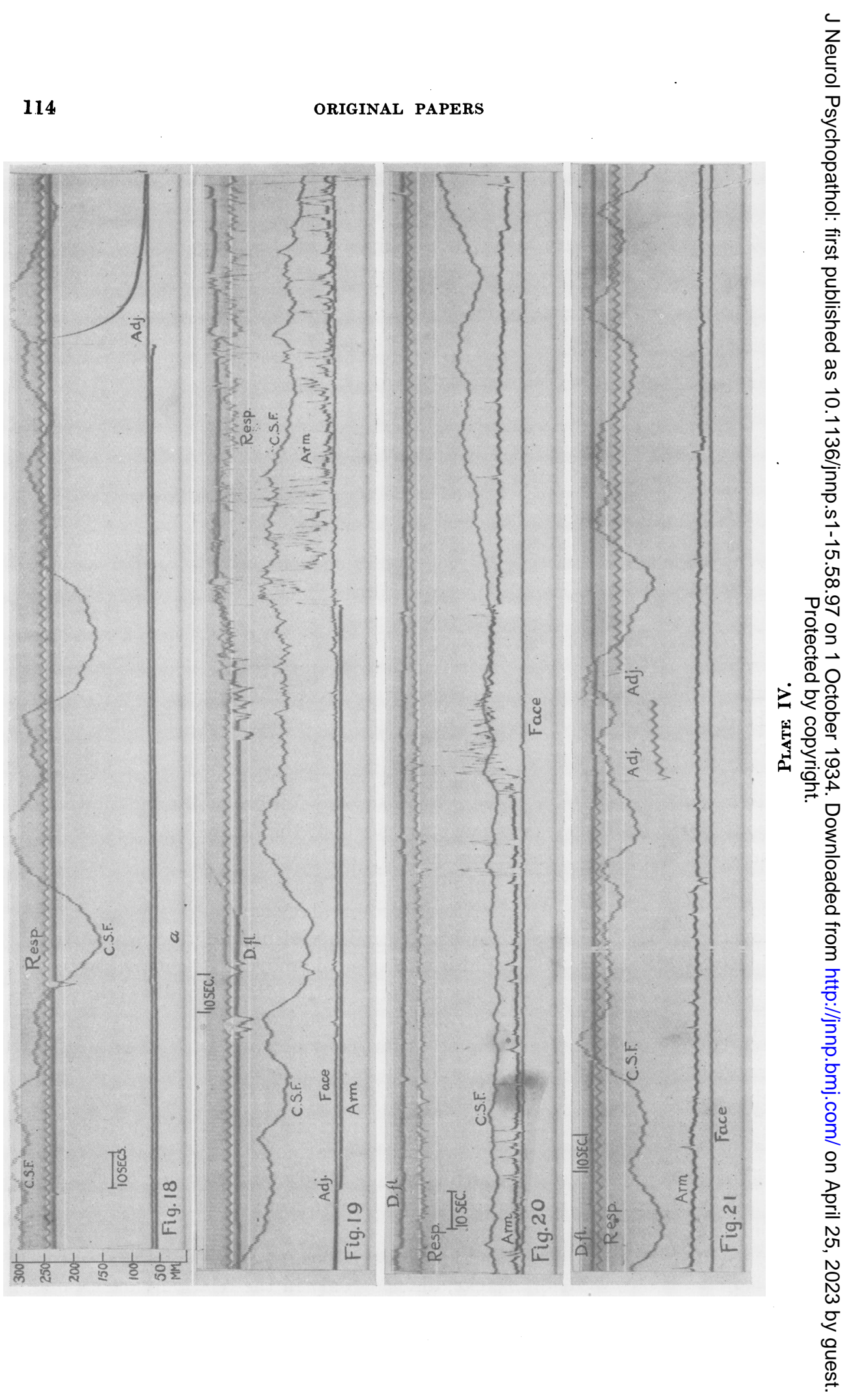


appeared spontaneously in the trough of some of the larger fluctuations in the drowsy state. Such a 'minimal fit' is shown in fig. 22. During the stuporose state stimulation, for example, by pricking the forearm, resulted in the gradual awakening of the patient with fall of the cerebrospinal fluid pressure in a series of wide fluctuations. When the pressure was low myoclonic twitching would commence abruptly, as in the spontaneous attack (fig. 17).

A loud clap of the hand during the drowsy state did not provoke myoclonus, although almost any stimulus increased the myoclonus when present. Inhalation of amyl nitrite during the height of an attack did not modify the cerebrospinal fluid pressure and affected the movements only in that they were slightly more violent for a brief interval. The rise of cerebrospinal fluid pressure during a fit appeared to be directly related only to movements of the chest and upper limb. Removal of 6 c.c. of cerebrospinal fluid did not modify the course of the attack. At the moment of removal the pressure was $240 \mathrm{~mm}$., the previous fluctuations being small and reaching to $200 \mathrm{~mm}$., the pressure after removal was $140 \mathrm{~mm}$., and rose after 20 seconds to $200 \mathrm{~mm}$., falling to $180 \mathrm{~mm}$. After removal of another 6 c.c. the pressure remained steady for 30 seconds at $100 \mathrm{~mm}$.

Case VI. Generalized convulsions at long intervals for seven years. For three years more frequent left-sided attacks, commencing in the left lower limb, without loss of consciousness. Continuous twitching of left foot for three months, with occasional spread to whole left side. Slight spastic weakness left lower limb. No organic cause found on cerebral exploration.

E. S., age 43 years, female, admitted under the care of Dr. Adie in March, 1934. No family history of epilepsy. Previous health good. Eleven years earlier, three months after the birth of her last child, she had a mild febrile illness during which there was an attack of unconsciousness with incontinence of urine. Twelve months later she again had an attack of unconsciousness with incontinence of urine. She was unable to say whether she had a generalized convulsion. From that time until three years before admission at long intervals similar attacks occurred. In one of these the tongue was bitten. In the last three yearr le attacks were more frequent, and more especially related to menstruation. In se without warning the toes of her left foot would become plantar-flexed, the lef $f^{.}$.er limb would be drawn up in a jerky manner, the left side of the abdomen and $r^{\circ}$ si would become involved, then the upper extremity

\section{DESGRIPTION OF PLATE IV}

Plate IV.-Figs. 18, 19, 20 and 21 (Case V) form one continuous record of the transition of events from sound sleep at the commencement of fig. 18, to drowsiness at the commencement of fig. 19, a prolonged myoclonic convulsion in the latter half of fig. 19 and the first part of fig. 20, and finally to sleep in the latter half of fig. 21. The cerebrospinal fluid tension (C.S.F.) for all figures is shown at the commencement of fig. 18. Movements of the foot, face and arm are shown by D.fl., Face and Arm as in fig. 16. At Adj. at the end of fig. 18 and the commencement of fig. 19 the tension of the arm balloon was adjusted but without disturbance of the patient, as was also the respiratory recorder at Adj. in fig. 21. 


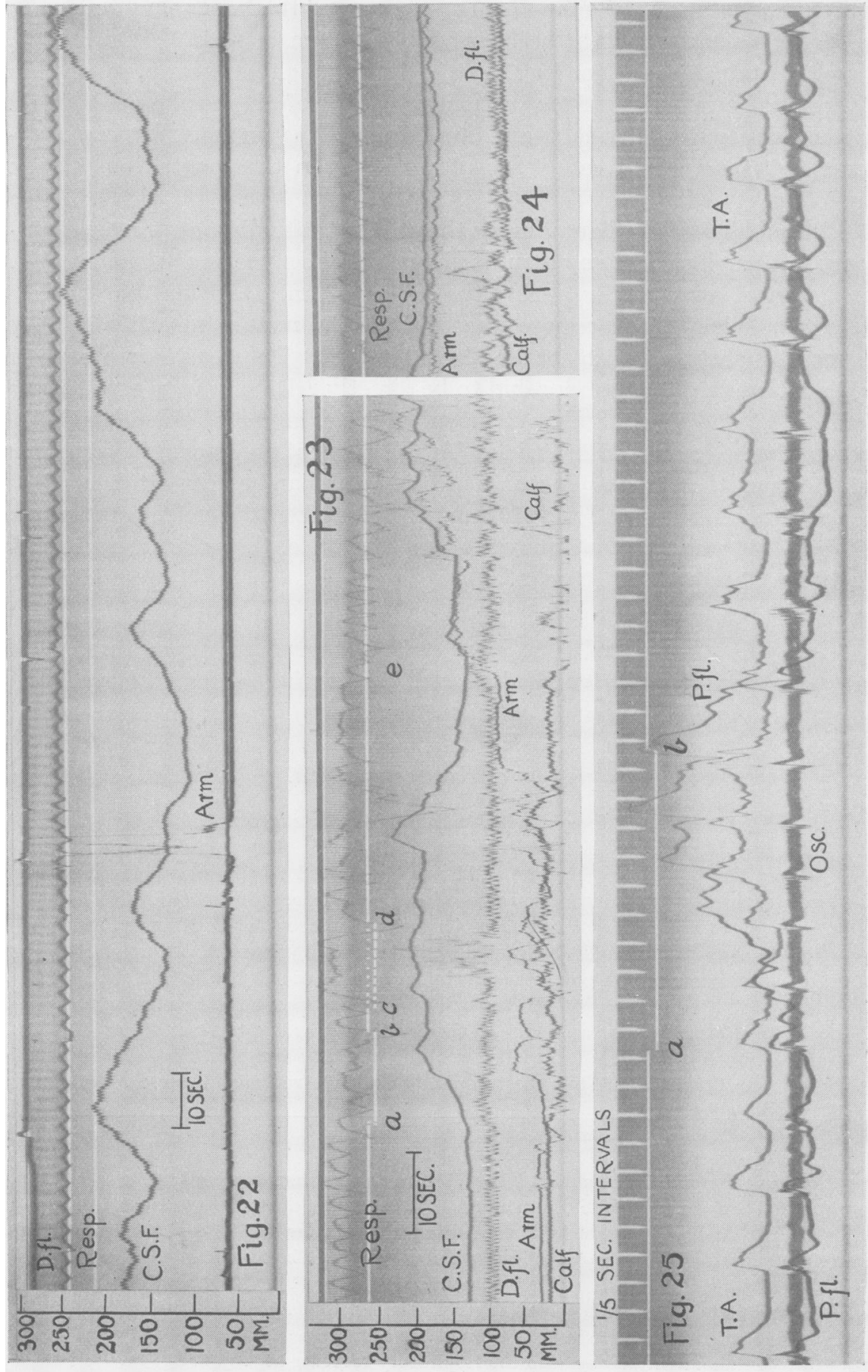

잉

혹

居

을

궁

호․

옳

음

음. 
and the left side of the face. With this type of attack, which lasted five to 10 minutes, there was no loss of consciousness. During these attacks all the fingers of the left hand were painful, as if ' broken off at the knuckles,' and following the sudden cessation of the attack, in all the parts involved she 'gradually felt the fingers coming back.' Occasionally a similar sensation was felt in the toes of her left foot. The right side was never affected. Commencing in July, 1933, there was an interval of four months without an attack. At the end of this time similar attacks recommenced at weekly intervals. In the three months before admission the left leg and foot were noticed to show irregular, almost constant, twitching, and the limb was felt to be weak on voluntary movement. Intermittent spread of the twitching from the left leg to the thigh, sometimes to the left upper arm, and more rarely to both left upper and lower limbs and the face, occurred. With this spread was associated a continuous drawing pain and numbness in the left leg as high as the knee and the left leg felt 'as though broken off at the ankle.' Medicinal treatment had been regular for three years.

\section{DESGRIPTION OF PLATE V}

Plate V. Fig. 22 (Case V).-C.S.F. pressure, respiration and movements of foot, arm and face, as in figs. 17 and 18 . To show a very small myoclonic convulsion chiefly affecting the arm, during partial emergence from sleep.

Fig. 23 (Case VI).-Record of plantar stimulation and resultant myoclonic activity with onset of myoclonic convulsion after a further interval. Beside the cerebrospinal fluid pressure (C.S.F.) there is recorded movement of the chest (Resp.), dorsiflexion of the foot (D.fl.) with upward movement for dorsiflexion, flexion of elbow (Arm) recorded by balloon over belly of biceps, and contraction of calf muscles (Calf) by small balloon adherent to gastrocnemius. At the beginning of the tracing a regular series of small movements of dorsiflexion of the foot are occurring; they had been present for some hours. As will be seen in later records their irregularity is at times interrupted by small pauses, at times by greater fusion of the twitches. In the interval indicated by the fall of the white signal line from $a$ to $b$ the sole of the left foot was stimulated with a blunt object causing some further tonic dorsiflexion of the foot and some general movement of the patient, with jerking of the arm record and irregularity of the respiratory record with rise of cerebrospinal fluid pressure. From $c$ to $d$ violent clonic movements of the foot, trunk and arm occurred which appeared to the observers to be involuntary in origin. These movements and the resulting irregularity in respiration rapidly settled down, but at $e$ the patient commenced to have a major convulsion during which she was unable to speak. This lasted some minutes and the resulting disturbance declined with gradual lessening of myoclonic twitchings, the termination of which is recorded in fig. 24.

Fig. 24 (Case VI).-The termination of the attack commencing in fig. 23. The alteration in the relative position of the movement records is due to change in posture of the arm and leg.

Fig. 25 (Case VI).--Record taken at faster rate to show the nature of the twitchings occurring at this time in both tibialis anticus and the calf muscles. Each twitch was of the nature of a slight plantar flexion of the foot passing immediately into dorsiflexion at the ankle with plantar flexion of the toes. The speed of the record is here increased so that the vertical white lines marking one-fifth seconds are here widely spaced. The horizontal thick record is that of the beam of a Matthews oscillograph (Osc.), which records the action currents in tibialis anticus. The upward displacement of the upper record (T.A.) reveals contraction of tibialis anticus, upward displacement of the lower record (D.fl.) reveals plantar flexion at the ankle. Each twitch of contraction in tibialis anticus is seen to be accompanied by an initial asynchronous series of small action currents. For the duration of the fall of the signal between $a$ and $b$ the patient is directed voluntarily to plantarflex the foot. 
On examination no abnormality was found in the cranial nerves or the upper limbs. There was slight spasticity in the left lower limb with some impairment of muscle power at all joints. The right lower limb was unaffected. The tendon jerks were increased in all limbs and more brisk in the left lower limb than in the right. The abdominal reflexes were equal and active, the left plantar response extensor, the right flexor. Sensation was normal except for a slight impairment of vibration sense over the left tibia. At times the peroneal muscles in the left lower limb, at others the flexors of the toes only, showed a fairly regular rapid series of twitches, which would continue for many hours. At irregular intervals the twitching would become more rapid and of wider amplitude and spread in the course of some seconds to involve the left thigh, left side of abdomen, left upper limb, and often the left side of the face. After two to three minutes the twitching would cease in the left side of the face and the left upper limb to remain in the toes or foot as before. Just after the subsidence or an attack pricking of any part of the left lower limb would again evoke spread to the left upper limb and face. On some occasions, but not in all, handling of the left lower limb during a quiet interval would be followed by a full spread of the attack. T. : same effect could be evoked by pin-pricks in this limb. There was no loss of consciousness in the attack. A right fronto-temporal surgical exploration revealed no surface change in the cerebral cortex. The attacks were continuing as before when the patient was last under observation early in June, 1934.

Analysis of attacks recorded on March 26 and May 7, 1934.-The cerebrospinal fluid pressure in this patient was $210 \mathrm{~mm}$., fluctuating between 262 and $280 \mathrm{~mm}$. as she fell asleep. During a period of observation of just over three hours there was continued irregular twitching of the tibialis anticus. For the greater part of the time no other muscles participated in this irregular twitching movement. The twitches were small and showed a general regularity in amplitude. When recorded by a balloon over the tendon of the muscle, connected to a small tambour manometer of high vibration frequency, they were found to take the form of groups of muscle twitches at times in small series of three to five, at times in partly fused pairs and often for long periods in a rhythm of single slightly longer waves at the rate of about two a second (fig. 23). 'There was no corresponding change in the cerebrospinal fluid pressure. At intervals of one to two hours the rate of twitching accelerated in tibialis anticus and at the same time twitching in the calf muscles, more proximal parts of the left lower limb and in the left arm occurred. The transition between the local attack and its generalization was gradual, as from $d$ to $e$ in fig. 23. The spread of the attack occurred in steps rather than in a gradual march. It was noticed that twitching of the calf muscles of minor kind would often accompany the twitching in the pretibial muscles for periods of several minutes without further spread of the attack. These continued twitches would often persist for many hours. On another occasion it was noticed that the twitching remained confined to the posterior tibial group of muscles alone for long periods. During the recorded observations the twitchings did not completely cease during sleep, but this was often observed in the ward.

The insertion of the lumbar puncture needle, the elicitation of a left plantar response, and pricking the left lower limb were observed to be followed 
by a generalized attack, but only when such an attack had not occurred for a long period. The result of scratching the sole of the left foot and the subsequent development of a widespread attack is shown in fig. 23. The plantar stimulation here evoked irregular twitching of the upper limb, and twitching continued in the calf, trunk and upper limb for some 20 seconds. With the irregular trunk movements the cerebrospinal fluid pressure rose unevenly and with their lessening fell to its original level. Within a few seconds twitching again occurred in all the parts concerned, becoming of very rapid vibratory kind : the attack having thus commenced continued irregularly for four minutes. The initial violence of the attack subsided somewhat after the first two minutes, and as respiration became more regular the cerebrospinal fluid pressure subsided more gradually, although the tension remained higher than before for many more minutes (fig. 24). There was no apparent loss of consciousness during the attacks. In this patient during a quiescent interval active hyperpnoa for a period of three and a half minutes did not evoke spread of the twitching which was at the time confined to tibialis anticus. A plantar response one minute later was followed by involvement of all the muscles of the lower limb in the twitching. A period of hyperpnœa lowered the cerebrospinal fluid pressure from 190 to $170 \mathrm{~mm}$., and recovery of pressure was delayed for a considerable interval.

On May 7, 1934, the twitching in tibialis anticus was recorded electromyographically together with mechanical records of the contraction of this muscle and of the calf muscles. The tetanic nature of each twitch and the way in which the twitching summated with voluntary movement and ankle clonus are discussed below.

\section{DISGUSSION}

It is evident that the recorded convulsions in these six patients differ considerably from patient to patient.

We were fortunate in having for these observations a wide range of phenomena, from the rapidly repetitive and very localized discharge of epilepsia partialis continua in Case VI to major attacks of sufficient violence to require the greatest perseverance in securing records, as in Case II. All patients with the exception of Case $V$ had at one time or another suffered from major convulsions, and we believe that the phenomena which they exhibit may be taken as fully representative of the effects of that process which underlies the symptom of epilepsy, and to which for brevity we shall refer as the epileptic process. We present reasons for the belief that this process was present in Case V. The myoclonus in these cases was of the more usual variety encountered in epilepsy, without familial characteristics.

It may be remarked in passing that the present cases serve to throw into prominence the artificiality of any very close identification of any particular variety of epilepsy with any particular variety of provocative pathology. The only rigid classification of epilepsy into organic and idiopathic categories is one of morbid anatomy. 


\section{The Cerebrospinal Fluid Pressure}

All observers are agreed that the cerebrospinal fluid pressure is increased during major epileptic convulsions, and pressures of $450 \mathrm{~mm}$. (Redlich and Pötzl ${ }^{24}$ ), $500 \mathrm{~mm}$. (Dalma ${ }^{4}$ ), $700 \mathrm{~mm}$. (Lennox and Cobb ${ }^{24}$ ), and $870 \mathrm{~mm}$. (Nawratzki and Arndt ${ }^{22}$ ) have been noted. Such changes in pressure might reasonably be expected as direct effects of the violent movements of major attacks. Ebaugh and Stevenson, ${ }^{7}$ by means of a tambour attached over a decompression opening in the skull, have observed a lowering of intracranial pressure for an interval of four and a half hours, covering three attacks of petit mal in one patient, while Elsberg ${ }^{8}$ has observed a rise of blood pressure and increased bulging of a decompression opening for six to eight hours before the Jacksonian attacks of one patient. Macdonald and Cobb ${ }^{18}$ have observed an initial fall of pressure preceding the onset of the convulsive stage in animals intoxicated with thujone. This initial fall of cerebrospinal fluid pressure, possibly related to the sudden pallor of the cerebral cortex occasionally observed by surgeons to precede a convulsion (Kennedy, ${ }^{15}$ Leriche ${ }^{17}$ ), accordingly has some more direct pathological interest.

It is evident from our records that abrupt change in the cerebrospinal fluid pressure may occur very early in the course of an epileptic fit. This change, which may be a fall or a rise, tends to a regular form in consecutive attacks in any one of the patients examined, and is much more obvious in Cases I, III, IV and VI than in Cases II and V.

Of the numerous factors which affect the cerebrospinal fluid pressure, the venous pressure is conspicuous in its rapidity and constancy of effect, characteristics which find common employment in the Queckenstedt and Ayer-Toby tests. The change in pressure engendered by light jugular compression as used in these tests is at first abrupt and rapid and then gradually reaches a steady plateau (fig. 4). The increase varies in height from patient to patient and according as the right or left jugular vein is compressed. Its mechanism may properly be related to distension of the cerebral veins, and its variability in extent to the anatomical variations of the drainage to each lateral sinus. The final plateau in this view indicates the full congestion of the cerebral veins drained by the sinus which is compressed.

The rudimentary valves of the jugular veins are well known to be incapable of obstruction of back pressure in this system so that from the right auricle pressure effects are directly transmitted to the cerebral veins. In such manner the rapid change in cerebrospinal fluid pressure early in a cough, as seen in fig. 9, receives explanation as a direct transmission of a wave of increased venous pressure from the thorax to the cerebral and spinal veins. When the patient is lying almost horizontal the cerebral veins are slightly distended so that not only is an increase of pressure transmitted from the thorax to the cerebrospinal fluid, but also any diminution of intrathoracic pressure. In this way the initial deep inspiration of the fit in Case IV, well seen in figs. 13, 14 and 15, accounts fully for the remarkably constant initial sharp 
fall of cerebrospinal fluid pressure in the attacks of this patient. So, too, close scrutiny of the record of chest movement in Cases I and III reveals a small irregularity, a small forced expiration, which accounts for the initial rise in these patients. The subsequent rise in the cerebrospinal fluid pressure is related to the period of apnœea, where the chest is fixed in expiratory spasm. The subsequent decline mirrors the gradual resumption of normal respiration which may be early after the termination of the attack as in fig. 1, or much delayed, as in figs. 12 and 24.

Rapid deep breathing causes a fall of cerebrospinal fluid pressure, as shown by Stevenson, Christensen and Wortis. ${ }^{30}$ No doubt the rapid fall in pressure to a low level following some fits (fig. 1) is the result of hyperventilation in the last stages of the attack.

In Cases II and IV, in which maintained change of pressure did not usually occur, the thorax and abdomen, though involved by myoclonic twitching, did not show inspiratory spasms (figs. $6,17,19$ ). Increased rapidity of respiration has but little effect, as at $b$ in fig. 12, and here later turning of the head with tonic contraction of the chest brings about a delayed rise.

The change in cerebrospinal fluid pressure in these recorded cases therefore reflects both the degree of expiratory spasm of the thorax and the time of that spasm in relation to the other phenomena of the attack. The relative constancy of form of the pressure change in any one patient is but evidence of the regularity of march of events in the attacks of that particular patient. The exact commencement of the convulsion is difficult to define. From records such as figs. 12,13 and 14 it is evident that some movement of the chest may precede the onset of convulsive phenomena in the limbs. Pollock and Treadway ${ }^{23}$ found changes in respiration preceding the convulsion in 12 out of 44 cases investigated, in the nature of an apnœa lasting 16 to 30 seconds, accompanied by fall in blood pressure. A similar affection of respiration possibly accounts fully for the fall in blood and cerebrospinal fluid pressure preceding thujone convulsions in animals. ${ }^{18}$ This change in respiration appears to us more properly regarded as an early convulsive phenomenon. In the preconvulsive stage we have observed a constant change in cerebrospinal fluid pressure in only one patient (Case V), a slow fall of pressure over many minutes, culminating in the onset of the convulsion. The mechanism of this type of preliminary change is discussed below.

\section{The Effect of Sleep}

Brodmann ${ }^{1}$ and Shephard, ${ }^{26,27}$ by means of a tambour applied over the site of cranial decompression openings, observed a rise of intracranial pressure during sleep, in contrast with the fall that Mosso ${ }^{20}$ had observed by a similar method. Stevenson, Christenson and Wortis, ${ }^{30}$ by continuous records of cerebrospinal fluid pressure by lumbar puncture, observed a rise of pressure during sleep. The amount of rise is not stated. In our Case V rise of pressure, with superimposed fluctuations, during sleep and drowsy 
states assumed most remarkable proportions (figs. 18 and 22), so that large waves of 100-150 mm. height and lasting over some minutes occurred. Such fluctuations were noted by Brodmann and Shephard in trephine openings and in drowsy states by Stevenson, Christensen and Wortis in the pressure by lumbar puncture. They have been identified with the well-known TraubeHering waves in the venous and arterial pressure, ${ }^{11,26,27}$ and their appearance in the cerebrospinal fluid is presumably a direct transmission via the cerebral veins. The great depth of fluctuation in Case $\mathrm{V}$ is possibly related to the presence of hydrocephalus as revealed by postmortem finding. In Cases I and II the fluctuations were slighter in degree but preserved the same characteristics (fig. 5).

In view of the increase of vascularity of the cerebrum which is necessarily entailed in these rises, it does not appear to us possible to maintain that sleep is related to cerebral anæmia. We have not observed any fluctuation in rate or amplitude of respiration accompanying these waves in the cerebrospinal fluid. Their origin is therefore related to some parallel fluctuation in pressure on the right side of the heart. Any explanation by periodic fatigue of the sympathetic centres ${ }^{30}$ or vasomotor centre ${ }^{11}$ appears to find more difficulty in explanation than fluctuations of tone in the trunk and limb musculature.

Whatever be the true explanation of this phenomenon the slow fall of cerebrospinal fluid pressure preceding the attacks in Case $\mathrm{V}$ is clearly the result of transition from sleeping to wakeful state (figs. 18-21). This transition no doubt plays a rôle in the circumstances predisposing to the onset of the fit, but cannot be termed an integral part of the attack.

\section{'Reflex' Epilepsy}

In four of the patients subjected to examination clinical observation of the frequent appearance of an attack shortly after some unusual stimulus had suggested the presence of ' reflex' epilepsy. This phenomenon, of which examples are described by Jack,son, ${ }^{13}$ Gowers, ${ }^{9}$ Wilson, ${ }^{32}$ and others, occurs in varying degrees. It has been described in very localized form such as in the case of Jackson, where a touch only on the face or head regularly elicited an attack. More commonly cases have been described where attacks may be regularly evoked by a sound. In both varieties the element of surprise appears to be necessary. Jackson states of his case that ' a fit would not occur if he knew he was going to be touched.' The provocative noise must be "a "startling" noise" (Gowers ${ }^{9}$ ), and of such a case Wilson ${ }^{32}$ notes "... aura and subsequent fit never arise "spontaneously" but always as a sequence to a sudden extrinsic stimulus of any kind provided it is sufficient to startle him.' To one of us a young man subject to fits immediately after any loud unexpected noise once explained that he had had an attack when a piece of paper lying nearby was unexpectedly turned over by a gust of wind, without any noise. It is also not uncommon to find that handling of 
the predominantly affected limb in a patient liable to focal epilepsy will occasionally set up an attack, as described by Gowers. ${ }^{9}$ In this variety of ' reflex' epilepsy, as we ourselves have observed, the manœuvre may be repeated many times without eliciting an attack, and it is only when sufficient interval has elapsed since a previous attack that the association can be demonstrated. This phenomenon has not in our experience been associated with 'acoustico-motor' epilepsy, but was seen by one of us in a patient previously liable to a right-sided attack when the right ear was touched.6

In the present series of cases the patient of Case IV during clinical examinations had seemed to commence an attack immediately following any stimulus to the affected limbs. During the periods that records were made the attacks occurred with remarkable regularity, and as has been explained in the protocol the records show no significant change of interval when a limb had been stimulated. The brevity of the intervals at the time of recording may have resulted in the disappearance of the phenomenon, but from our records we have to regard the apparent association of stimulus and fit in this patient as probably a coincidence. In Case II attacks were more regularly elicited by noise or other surprise, and in Case VI by handling or stimulation of the affected lower limb. The attack arose at an interval from the stimulus (figs. $7,17,23$ ) and differed in no way from those which arose spontaneously. The interval between stimulus and attack was, however, most interesting. In both patients the stimulus aroused an immediate outburst of myoclonic twitching. Such jerking is well known clinically to be provoked by surprise or start, as the studies of Pierce Clark ${ }^{2}$ and Muskens ${ }^{21}$ especially emphasize. Stimuli such as a scratch to the sole of the foot may be expected to increase local reflex excitability, and we would attribute the enhancement of the twitching in the foot by such a stimulus in Case VI (fig. 23) to such a process. If the cortical epileptic discharge may be regarded as having widespread subthreshold effects on spinal neurones other than those provoked to actual discharge, the ' reflex' enhancement becomes the summation of two stimuli, one reflex, one epileptic, on the common final path.

In Case $\mathrm{V}$ the occasional appearance of myoclonus during the troughs of the waves of cerebrospinal fluid pressure (fig. 22) may be taken as evidence of direct association of the waking state and such epilepsy. It is therefore further possible to interpret the epileptogenous effect of such a stimulus as pricking in the same patient (fig. 17) as an indirect result mediated by greater wakefulness. The long delay in appearance of the fit and the falling cerebrospinal fluid pressure document the process of awakening, to which the term ' reflex' cannot with accuracy be applied.

It appears to us that the process of myoclonic stimulation in waking states, such as recorded in fig. 7 , has only quantitative difference. The patient is awakened from a state of partial wakefulness to one of greater wakefulness.

To align the phenomena of ' reflex' epilepsy with the known charac- 
teristics of spinal reflexes it appears to us to be necessary to abandon the notion that the fundamental epileptic process is in some way part of a reflex arc and to substitute the alternative hypothesis that the fundamental epileptic process is continuous and widespread, and in its lesser intensities productive of outward effect only by coalition with other intense nervous processes. In this view the effect of an added stimulus is related solely to its power to cause a sudden increase in excitability in the patient concerned. In Case VI the spasticity and heightened flexion reflex in the left lower limb as a result of a cerebral lesion not only enable the enhancement of attacks by stimuli to that limb, but possibly may be held to contain the explanation of epilepsia partialis continua in that limb for many months. The early pallor with fall of blood pressure in some generalized epileptic attacks, and particularly (though not constantly) in petit mal, and the early apnœa we have recorded, may in this view be but the first evidence of the rise of generalized epileptic excitation, reflected in nervous mechanisms already active, and hence more sensitive in revealing change. It is immaterial that the neural levels of these mechanisms are the lowest, for the rising epileptic excitation is passed on to all levels, or conversely a cortical representation may be urged for all. ${ }^{9}$

Sudden 'starts' can be elicited from the thalamic animal by a loud sound. Muskens ${ }^{21}$ claims that similar starts have a reflex mechanism in the pons, and identifies them with the myoclonic twitch of epilepsy. He has widely investigated the enhancement of this reflex by monobromide of camphor. We would urge that the start reflex be regarded as a true reflex distinct from the epileptic state. Epileptic myoclonic twitching exists in patients in whom the reflex start to surprising stimuli is not present, and in them auditory stimuli do not appear to provoke attacks. Where the start reflex is readily obtained in an epileptic patient, as in our Case II, a sudden jerk in the flexors of the limbs is the immediate consequence of an unexpected sound. The jerk or 'start' facilitates an outburst of myoclonic twitching, and whether or not an epileptic fit develops appears to depend on the coincident condition of the 'epileptic state.' In this way the effects of wakefulness, spinal reflex, and reflex 'start' may be brought into the category of facilitation at the final common pathway. Whether the site of convergence of these effects be cortical, or pontine, or spinal probably differs from case to case. On general grounds it is our belief the common site is cortical, some reasons for which will be discussed below.

\section{The Incoordination of the Epileptic Discharge}

It has been maintained as a result of cinematographic analysis of epileptic convulsions (Stenvers ${ }^{29}$ ) that the movements are sequences of coordinated single movements such as the neck reflexes of Magnus. Martin ${ }^{19}$ describes the movements of the legs as spinal reflexes similar to the walking reflexes of experimental animals. Such movements should be highly organised, so 
that there is essential harmony of contraction in each phase, and they should be completely integrated.

In our records of epileptic myoclonus and the phenomenon of epilepsia partialis continua there is frequent evidence of complete irregularity both in time and place, and we feel that this lack of organization is of importance not only in these minor convulsive phenomena but in all degrees of epileptic convulsion. The biting of the tongue is clear expression of this fundamental incoordination. In this we find full accord with the definition of epileptic discharge as a ' discord of movement' (Gordon Holmes ${ }^{10}$ ), and the statement of Kinnier Wilson ${ }^{32}$ that the outward 'caricatured movements in no way resemble those called voluntary.' The sole coordinated motor discharges of epilepsy are the behaviour phenomena of the aura and of automatism which are not proper to the convulsive stage with which we are here alone concerned.

The essential irregularity of the myoclonic convulsion is seen in figs. 8 and 16, where various groups of muscles enter haphazard contraction in irregular time relation. Records of the twitching in Case VI (figs. 24-29) appear of particular interest in this connexion, for here long continued cortical discharge excited flexors and extensors concurrently, and brought a twinge of pain into each momentary paroxysm. The foot was contorted in meaningless fashion. In the records of major attacks in other patients the convulsion of the chest not only made abrupt cessation of respiration, but its incoordination with the movements of the glottis produced the 'epileptic cry.'

Case VI was also particularly suitable for the exploration of the relationship between voluntary and other movement and the intermittent paroxysms. A Matthews oscillograph gave us particularly clear records of the nature of the discharge. In the tibialis anticus this took the form of outbursts, each lasting usually one-fortieth of a second, and composed of six to 11 rapid galvanometer vibrations, each corresponding to a volley of impulses (fig. 25). The resulting twitch of the muscle thus appears to be in the nature of a brief motor tetanus. The intervals between the outbursts are 'silent,' and no discharge reaches the muscle. This discharge differs from reflex spinal clonus in the scattering of the discharged impulses, for in the latter there is synchronous or almost synchronous discharge of one volley for each wave of muscular contraction.

Voluntary movement had no effect upon this discharge. During voluntary plantar flexion the discharge continued as before (fig. 25). Voluntary dorsiflexion of the foot added a normal continuous series of nerve impulses in rapid sequence to the epileptic discharge, so that if the movement were powerful the epileptic discharge was almost swamped (fig. 27), while with weaker efforts the epileptic impulses were less covered (fig. 28).

At intervals each outburst of discharge increased in duration and the intervals lengthened. This change occurred either as the occasional appearance of an isolated longer outburst of one-tenth second duration, or by the: 


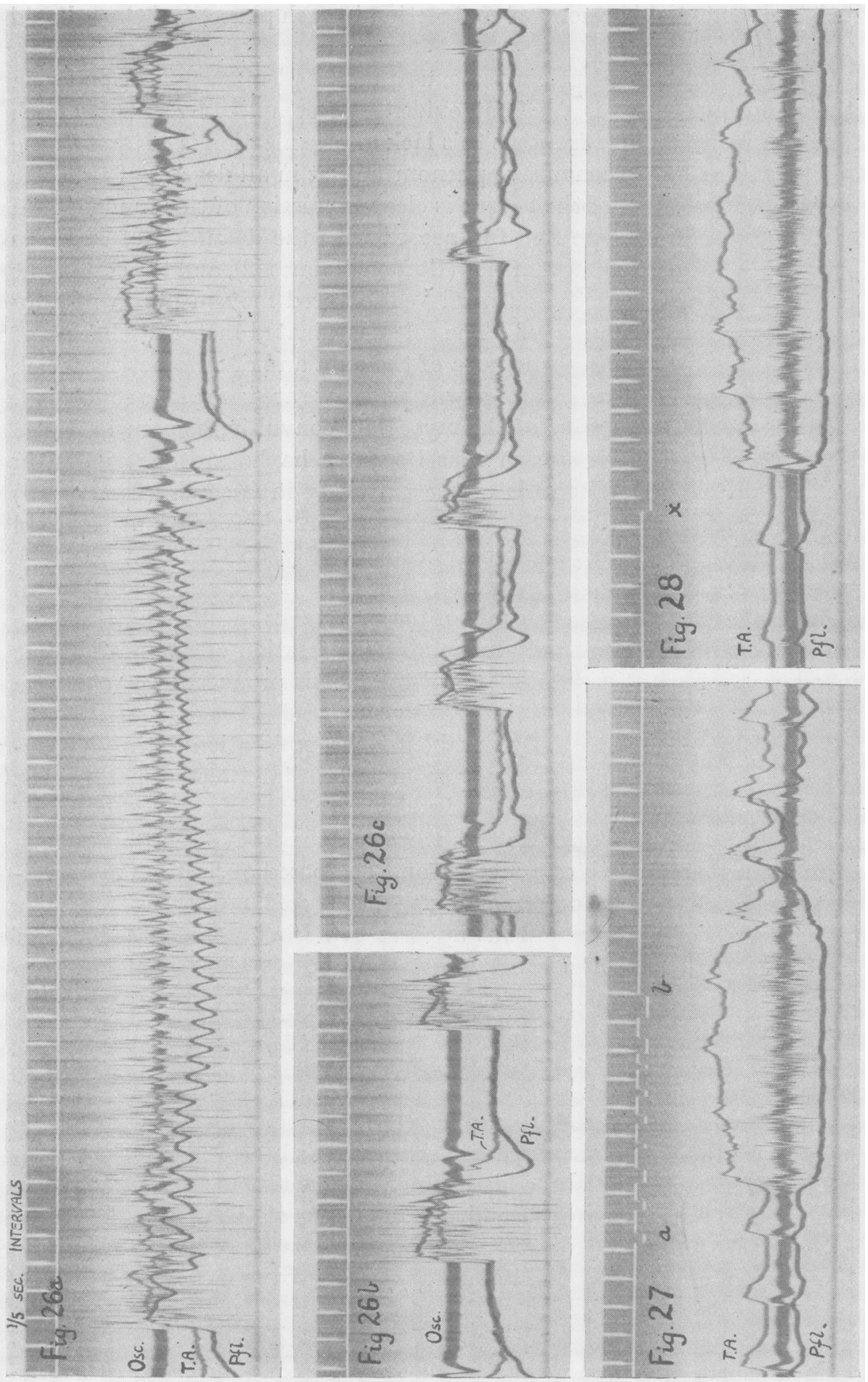


progressive lengthening of discharge to separate continuous outbursts of tetanic discharge, each lasting up to 10 seconds. An example of the latter type is shown in fig. 26a, and the progressive shortening which characterized the return to the more usual rhythm. The prolonged outburst is seen to be essentially clonic in nature, with frequency increasing from eight to 13 beats a second. The corresponding muscular contraction is a partially fused tetanus, and still involves both the opposing dorsiflexors and plantar flexors of the ankle. In both brief and prolonged bouts of discharge a contraction of the plantar flexors of the ankle preceded the contraction of tibialis anticus by a brief interval, tending to lessen as the contraction of tibialis anticus increased. Following a series of such spasms of increased duration the usual brief attacks either were much diminished in amplitude or disappeared entirely for some minutes. In the major attack in this patient, shown in figs. 23 and 24, this type of discharge does not appear, though the duration of each twitch is longer during the attack than before, especially in the partial attack elicited by stimulation of the sole of the foot (fig. 23, c-d). We do not regard such maintained clonic outbursts as different from the more irregular variety recorded in spontaneous major attacks. Both were invariably accompanied by spread of the epilepsy to other muscles. Prolongation of discharge by change in rhythm is therefore one aspect of increasing activity of the epileptic process.

It was readily possible in this patient to elicit ankle clonus in the affected limb. In fig. 29 is shown the effect of combining the local reflex rhythm of ankle clonus with the rhythmical epileptic discharge both of the discrete brief type and of the more prolonged type. The transition in type of discharge is here possibly in part due to the handling of the limb, such transitions being often found under these circumstances. As with voluntary movement, the rhythmical cortical discharge continued throughout the duration of induced ankle clonus, and affected the latter in two ways. The heightened tone of the calf muscles was diminished during each twitch of tibialis anticus, and the rhythm of the ankle clonus was reset by each epileptic outburst

\section{DESGRIPTION OF PLATE VI}

Plate VI. Fig. 26 (Case VI).-Records as in fig. 25 to show the nature of long-maintained clonic attacks which occasionally arose spontaneously in this patient. Fig. 26a is an example of a long-maintained tonic attack, and the gradual resumption of the more usual smaller attack at the end of fig. 26c. Fig. 26b is the direct continuation of fig. 26a, while fig. 26c followed fig. $26 \mathrm{~b}$ at an interval of $3 \frac{2}{5}$ seconds. The single slow wave in the electromyogram after the termination of each attack is due to passive movement of the galvanometer leads.

Fig. 27 (Case VI).-As figs. 25 and 26. To show the effect of strong voluntary dorsiflexion of the foot which the patient was ordered to perform in the duration of the interruptions of the signal between $a$ and $b$.

Fig. 28 (Case VI).-As fig. 27, a few seconds later in the experiment. At $x$ the patient was ordered to make a slight voluntary dorsiflexion of the foot. 


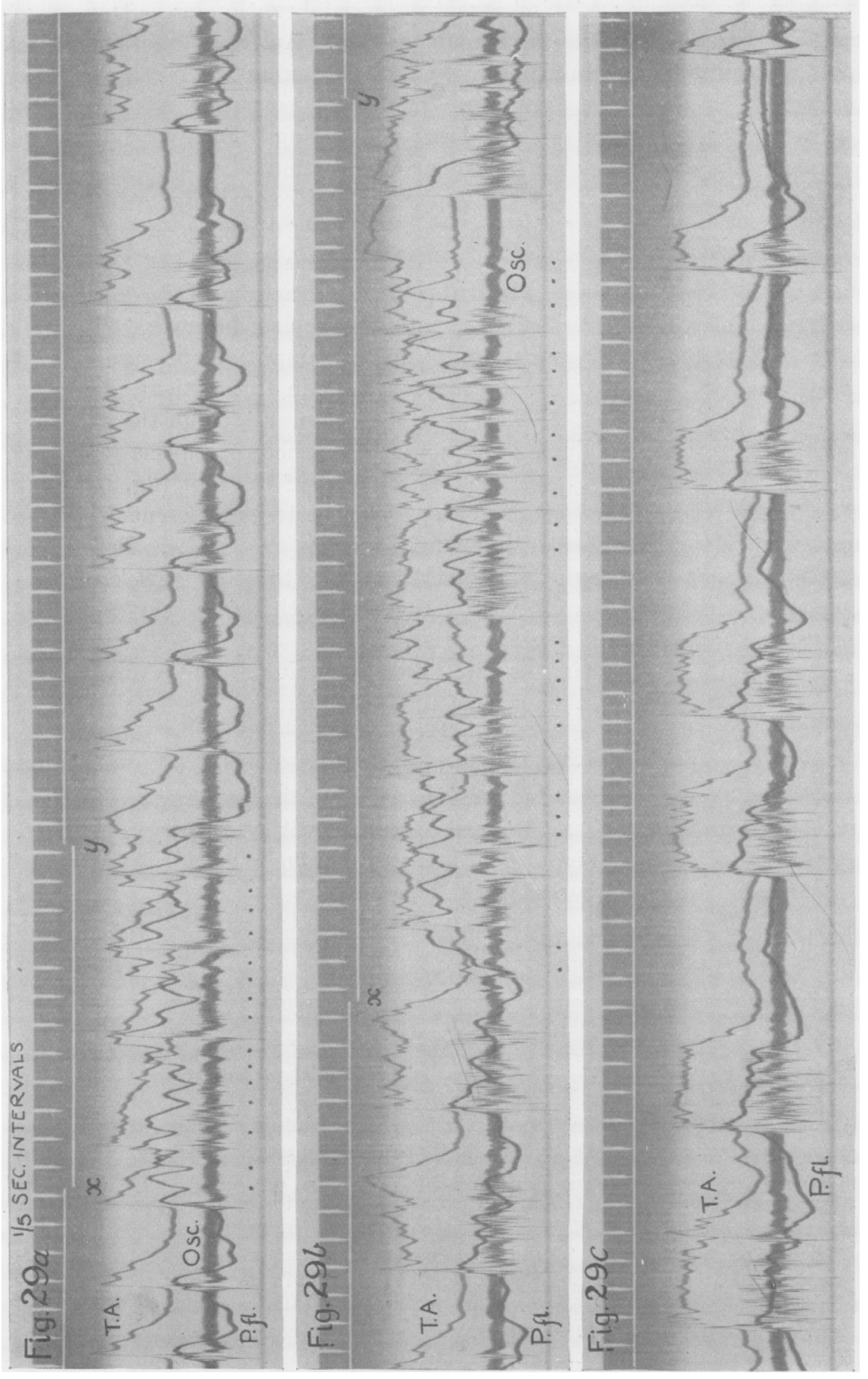


(fig. 29a). When the epileptic discharge is more prolonged the rhythm of ankle clonus apparently combines with the rhythm of cortical clonus, and in tibialis anticus the epileptic action currents assume combinations between ' cortical' rhythm and the reflex rhythm of its opposing muscular group (fig. 29b).

The cortical discharge of epilepsy is therefore independent both of voluntary movement and of spinal reflexes, though both combinations sum their effects in the final common pathway. There being no certain evidence that the epileptic discharge is transmitted by pathways other than the pyramidal tract, we may provisionally assume that the locus of convergence of voluntary and epileptic innervation is on the dendritic expansions of the Betz cells in the motor cortex.

In Cases II and V myoclonic twitching actuated each muscle for short periods in brief attacks, and in records of movement such as fig. 16 the character of the discharge in any one muscle differs not at all from that occurring more regularly in the muscles of Case VI. In the former patients the epileptic process has multiple foci of fluctuation; in the latter a single focus in the leg area varies in the spread of its effect. Whether the convulsion be particulate as in myoclonus, or diffuse as in the major attack, incoordination of the cortical discharge is its essential feature. It reflects a global stimulation of a cortical area foreign to the selective niceties of voluntary movement.

Some degree of concurrent contraction of the opposing flexor and extensor muscle groups at a joint is not uncommon in the course of spinal reflexes. The phenomenon most commonly arises in the antagonism between two contrary reflexes of similar potency and has explanation in the process of 'double reciprocal innervation.' 28 Its analysis in the laboratory animal demonstrated clearly that here the spinal neural mechanism preserves a regulated relative balance between opponents, so that the law of reciprocal innervation is not in fact upset. In more complex posturing variable degrees of concurrent innervation of opponents are also found, and for reflex movement

\section{DESCRIPTION OF PLATE VII}

Plate VII. Fig. 29 (Case VI).-Records of contraction of tibialis anticus (T.A.) and plantar flexion of the foot (P.fl.) with electromyogram of tibialis anticus, as in figs. 25 to 28. The more usual small epileptic twitching had continued uninterrupted for some minutes before this record. In fig. 29a three such beats occur when at $x$ an observer by passive dorsiflexion of the ankle elicits ankle clonus. The individual beats of the clonus are seen in the record P.fl. and the time of onset of each is further indicated by the black dots, which have been placed below the record. Following the termination of the ankle clonus at $y$ the epileptic twitching continues with prolongation of the beats and the intervals between beats.

Record in fig. $29 \mathrm{~b}$ commences after the lapse of some 10 seconds during which there was no material change in the discharge. At $x$ ankle clonus is again elicited by an observer and is discontinued two-fifths sec. before $y$.

Fig. 29c shows the course of events in direct continuation of fig. $29 \mathrm{~b}$. 
it is the invariable rule that for each moiety of contraction exerted in the prime movers there is an opposite inhibition of the opposing muscle centre. The more rapid the movement the more effective is the counter inhibition.

Voluntary movement in man possesses in its synergy readily verifiable co-contraction of antagonists, a feature which has been maintained by some to invalidate the hypothesis of reciprocal innervation. Synergic concurrent contraction has, however, another aspect. The difficulty in overcoming the spasm of cramp, or the burden of spasticity, by voluntary movement is sufficient disability to make it clear that the contraction of an antagonist most definitely requires limitation. It is a philosophical necessity to enquire whence this limitation, which ensures the precision and rapidity of voluntary movement, is derived. It appears to us to require the most exquisite final development of that process of regulation of antagonists which the principle of reciprocal innervation defines.

The convulsions of asphyxia or of intoxication by strychnine in the spinal animal are composed of alternations of rapid scratching, running, leaping, vomiting, each well coordinated and of regular though rapid rhythm.

Movements resulting from moderate electrical stimulation of a motor point in the cerebral cortex possess outward show of coordination, probably mediated by the spinal motor mechanism. Immediately the stimulus is removed a sudden damping of all discharge in all muscles is often followed by a powerful outburst of contraction unlike the primary movement. Alternating outbursts of this after-contraction form the well-known epilepsy resulting from cortical stimulation in animals, a phenomenon which at times marches from muscle to muscle. Examined myographically, the individual spasms of this 'clonus' are at times reciprocal in opposing muscles, but at others are concurrent, producing an incoordination so powerful that double reciprocal innervation no longer accounts for it. ${ }^{3}$ This incoordination of the ' after-discharge' agrees with other evidence that the electrically produced movement is itself also the outcome of a mixture of effects in which outward coordination appears to be the added approximation of reciprocation by the spinal mechanism. However small the electrode, a stimulus which excites the motor cortex at all excites a mosaic of units of differing and opposing functional sign. ${ }^{3}$ Clonic after-discharge is the rhythmical dissipation of incompatible residues.

Both in the records of epileptic clonus here described and in the epileptiform after-clonus from cortical stimulation the tetanic nature of each individual outburst, the complete silence between outbursts, and the lack of reciprocal coordination serve to identify the same underlying mechanism of production. The clonic spinal stretch reflex (spinal clonus) is by complete contrast a series of synchronous volleys of single nerve impulses. These differences between convulsive clonus and spinal clonus render it extremely unlikely that the former is a release of spinal reflexes by the removal of 
cortical inhibition. We have already remarked that lack of reciprocal innervation in convulsive discharge has only a quantitative difference from that which is traceable in the result of unipolar stimulation of the cortex. Both represent the global stimulation of a microscopical mosaic. Conversely the property of activation of single movements, or even single muscles, is common to both, and reflects the stimulation of relative concentrations of units of like function from a general anatomical pattern unlike any other in the nervous system. A movement limited to a single joint (cf. Cases V and VI) stamps its origin from the motor cortex as much for epilepsy as for electrical stimulation. In the myoclonic variety of epilepsy such isolated movements (figs. 8 and 16) may be watched for hours on end, and their local character completely denies any pontobulbar localization of their origin.

We therefore conclude that the discharge of an epileptic convulsion may be more truly likened to a discord of the units of movement, a discord without component melodies, so that it appears not strictly accurate to regard a convulsive movement as a 'contention of very many of the patient's familiar normal movements.' 14 The natural organization of voluntary movement has a selective mechanism which the convulsion ignores.

\section{The Periodicity of Seizures}

Periodicity of the motor discharge of epilepsy is present in many degrees. Not only is it found in the recurring twitches of epilepsia partialis continua and in myoclonus, but it is also present as the rapid clonic discharge which makes up longer outbursts. Besides these rhythmical phenomena in the single convulsion there has also long been recognized a tendency in some patients for fits to occur in groups, each group of as many as 20 fits being separated from similar groups by regular intervals (Gowers ${ }^{9}$ ). Status epilepticus occurs most usually as an isolated event, but in Cases II and IV the periodicity of its development was particularly well defined. In the former the frequency of attacks in three such outbursts at intervals of nine months and eight months is plotted in fig. 30. Although the phenomenon rarely occurs in such perfect form, minor variants, such as is outlined in the history of Case III, are not infrequently encountered. The phenomenon is as much a property of 'idiopathic' epilepsy as of epilepsy of organic origin (Case IV). When the number of attacks from day to day in Case II are charted the regularity of accrescence and decrescence in number of attacks is even more striking, for, although the amount of treatment had been increased shortly before the peak of the attack, the smoothness of rise and decline, in spite of abrupt changes in treatment, gives evidence of a process of fluctuation not related to treatment. Portion of such a decline is also seen in the figures given for the frequency of observed attacks in Case III. Though at first glance the actual turn or peak of the graph appears wholly referable to the intensity of administration of sedatives in various forms, this interpretation fails to account for the absence of effect of heavy dosage in the 


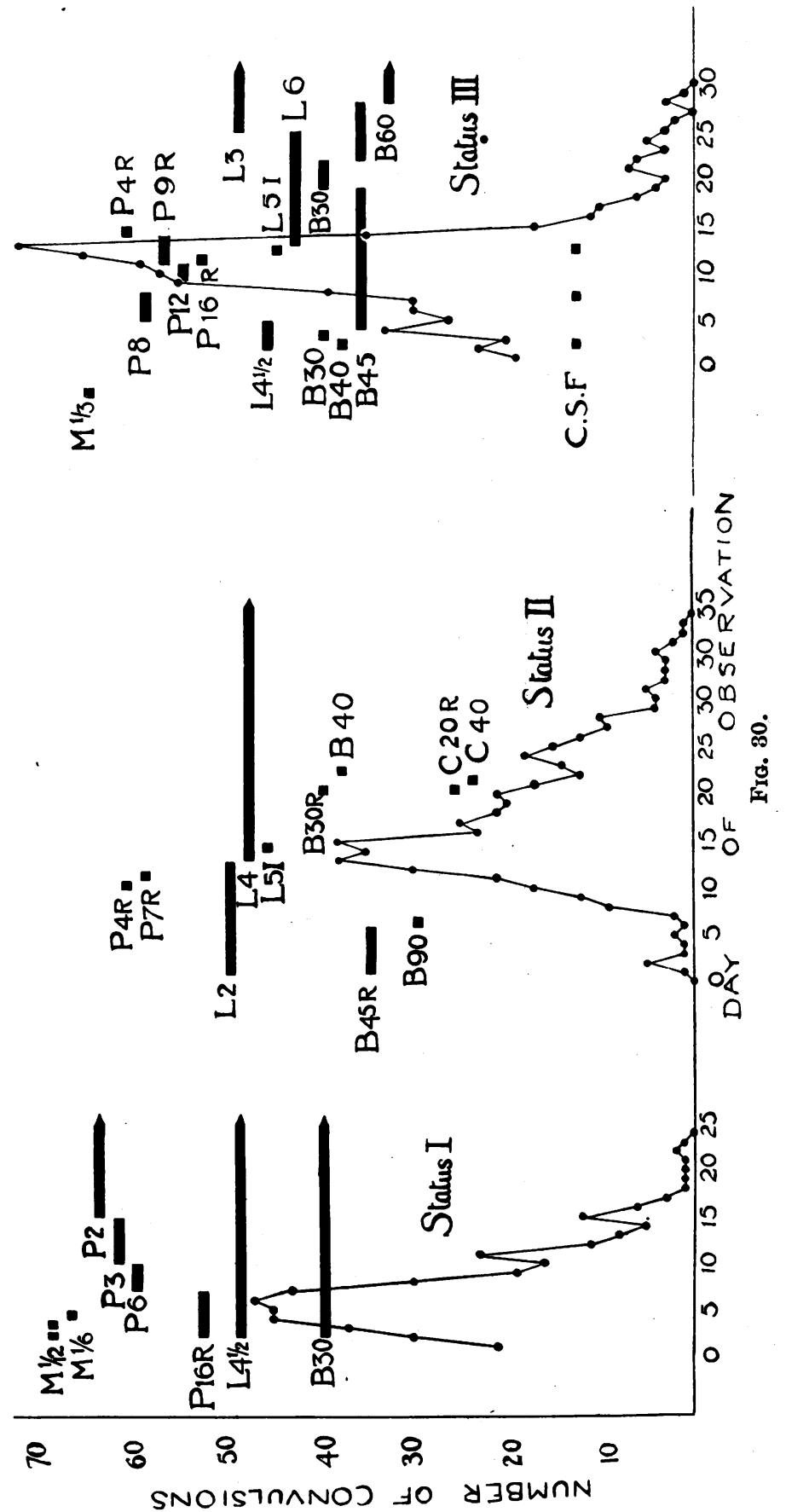


early stages in status III (fig. 30), or the final termination in diminishing series of few attacks in all three states'. Indeed the initiation of a succession of attacks during regular treatment is strong evidence of some fluctuation in the underlying process. The form of the curves finds more ample interpretation as the expression of a natural evolution and involution deeper than the effect of treatment. Our Cases II, III and IV presented each the wellknown transitory postepileptic hemiplegia of Todd ${ }^{31}$ and Robertson. ${ }^{25}$ In this condition the inevitably recurring attacks continue in limbs which may be early completely paralysed for all voluntary movement. In the intervals between convulsions they lie flaccid and inert. Though we have not made records of attacks in the same limbs before and after the appearance of this exhaustion, we have observed, especially in Case II, no essential difference apart from a shortening of duration of attack, a fact which is most difficult to reconcile with any of the theories of epilepsy as a reflex, or ' short circuiting,' or removal of inhibition, or indeed the explosive theory, all so fully discussed by Lennox and Cobb ${ }^{16}$ in their monograph.

The time of onset and the time of complete development of postepileptic hemiplegia are not directly related to the commencement of decline in the numerical frequency of attacks, nor is any recurrence of fits referable to full recovery from the paralysis. The maintenance of the character of the attack during the paralysis further suggests that the phenomena have different anatomical loci. Neurones which cannot be voluntarily excited for periods of weeks continue to be available for the gross cortical discharge of the convulsions. The epileptic process is so situated that it may affect the mechanism of voluntary movement without impairing the spinal reflexes, for the tendon jerk, valuable indication of the state of the spinal reflex arcs, may remain unaffected. The flaccidity appears identical with that consequent upon lesions of the parietal cortex. Flaccidity of the affected limbs is by no means the rule, and variable degrees of spasticity may be present, even varying from one bout to another in the one patient, as in Case II. The phenomenon may in our experience affect cortical sensation as much as voluntary

Fig. 30.-Graphic representation of the number of attacks per day in Case II, in each of the three periods of status epilepticus in which this patient came under hospital observation. The number of attacks per day is recorded for each successive day covering 25 days from August 4, 1931 (status I), 35 days from May 18, 1932 (status II), and 30 days from January 2, 1933 (status III). Horizontal black lines indicate the duration of each of various forms of treatment. The lettering with each horizontal line records the form of treatment with the dosage in grains in each 24 hours; thus M. $\frac{1}{3}$ represents morphia gr. $\frac{1}{3}$ in 24 hours, $P$. represents paraldehyde with the number of drams by mouth in each 24 hours and where given per rectum the dosage is followed by the letter $R$. Phenobarbital is represented by the letter $L$, and where on two occasions 5 grains of phenobarbital sodium was given intravenously, this was represented by $L 5$ I. Bromide by mouth is represented by $B$ with the number of grains in 24 hours. Chloral by the letter $C$ with the dosage in grains in each 24 hours. On three occasions cerebrospinal fluid was withdrawn in quantities of 30,36 and 30 c.c., represented by the squares after the letters C.S.F. 
power (Cases II and IV). It is to he sharply distinguished on the one hand from the progressive hemiplegia resulting from an organic lesion as in Case I, and on the other from transitory loss of power when that occurs as a feature of isolated convulsions, as in Cases I and VI (' inhibitory epilepsy'). True postepileptic hemiplegia develops slowly after frequently repeated attacks. Those parts 'which have been first and most convulsed are those left paralysed' (Jackson ${ }^{12}$ ).

We have invoked the principle of subliminal (i.e. subthreshold) central effects to derive an explanation of 'reflex' epilepsy. In that case two processes, each alone ineffective, may combine to throw neurones on which they converge into discharge. It appears to be a logical corollary that any raising of threshold at the site of convergence may exclude weaker effects while still allowing the excitation by stronger. It is a general physiological principle that fatigue raises threshold, and the exhaustion of the outgoing neurones of the motor area by frequent epilepsy may reasonably be expected to result in a raised threshold to all synaptic convergence upon them. An epileptic convulsion may be likened to intense electrical stimulation of the motor area, so that it may remain effective when it has excluded voluntary movement by fatigue. A state of exhaustion which may persist for many days after the subsidence of the epileptic discharges appears a necessary postulate and was recognized by Todd, Robertson, Jackson, and others. Its relationship to the occurrence of an extensor plantar response for some minutes after each attack (cf. Cases II and III) is difficult to define. Provisionally it would appear necessary to regard both as aspects of the same phenomenon, but there is as yet no evidence that reflex changes of this kind are unrelated to persistent cortical after-effects other than exhaustion. In Case VI, myoclonic epilepsy in the right side disappeared with the onset of a permanent hemiplegia, but here widespread cortical disintegration was proceeding with great rapidity. An irregular high temperature accompanied the syphilitic encephalitis. In the other patients slight irregular temperatures up to $99 \cdot 8^{\circ} \mathrm{F}$. were common. We did not encounter in this series of cases the high temperatures of the more grave forms of status epilepticus.

The periodicity of epilepsy has two aspects, namely the interval between one attack and another, and the intervals between groups of attacks. The declining frequency of attacks as the grouped epilepsy passes off is often unaccompanied by exhaustion paralysis, just as there may be complete absence of impairment of voluntary innervation in any part of the intervals between attacks. These intervals must therefore be related to a variety of exhaustion in neurones other than the pyramidal tract and spinal motoneurones. The convulsive process overflows in discharge, while the means of its renewal, a fundamental epileptic process with longer rhythm of fluctuation, appears to be necessary to give rise to such periodic outbursts as grouped epilepsy or periodic status epilepticus.

Epilepsia partialis continua would appear to offer an example where 
all the cortical processes of epilepsy are related to a well-defined group of cortical neurones, the motor area of the part concerned. In Case VI our analysis finds the pathway for motor innervation unaltered by the regularly recurring paroxysm, so that here with the simplest possible mechanism the course of the attack may be stated to originate beyond the mechanism of excitation and conduction in the Betz cells. There is no anatomical structure or physiological mechanism which can be identified with the locus of the fundamental epileptic state. It may be local or widespread in effect, and it may undergo fluctuation as a whole, or in most isolated subdivision such as we imagine to occur in myoclonic twitchings. Its effect on the motor cortex most closely resembles the diffuse stimulation of areas of variable extent.

While it may appear banal to reflect that the high proportion of fatal terminations of status epilepticus is evidence of exhaustion of the patient before exhaustion of the epileptogenous state, it nevertheless appears of the utmost importance that the rationale of treatment of the condition should not be the suppression of attacks at all costs, for in many patients this is not possible by any remedy other than a continuous general anæsthetic which appears to rest the epileptic process as much as, if not more than, the patient. A primary object of all treatment should be the adequate nourishment of the patient, combined with the maximum of sedative which is not otherwise injurious.

\section{GONGLUSIONS}

1. During epileptic convulsions the pressure of the cerebrospinal fluid shows changes reflecting passively the fluctuations of venous pressure arising chiefly from involvement of the thorax in the convulsion.

2. The cerebrospinal fluid pressure rises in sleep, and there is fluctuation in drowsy states. A slow fall of pressure preceding a convulsion is evidence only of an awakening of the patient.

3. Certain phenomena such as awakening, starts, or even local spinal reflexes, may enhance the tendency to epilepsy and thus appear to be the direct excitant of discharge. Such effects when they occur are examples only of the addition of one nervous excitation to another.

4. The convulsive stage of the epileptic attack is essentially an incoordinated discord resulting from the diffuse excitation of outgoing cortical neurones. Even in the most localized forms of epilepsy the essential epileptic process does not appear to be directly located in these neurones.

5. The exhaustion of nervous activity underlying postepileptic hemiplegia is related to threshold changes in the excitability of pyramidal neurones, whereas the intervals between attacks and the fluctuations in tendency to attacks are related to an unphysiological mechanism which has no defined anatomical locus.

We wish to acknowledge our indebtedness to the Medical Research Council for the use of myographic recording apparatus granted to one of us 
(D. D.-B.), and to the various members of the Honorary Staff of the National Hospital for permission to investigate patients under their care.

\section{REFERENGES}

1 Brodmann, K., Jour.f. Psych. u. Neurol., 1902-3, 1, 10.

2 Clark, L. Pierce, Jour. Nerv. Ment. Dis., 1919, 50, 17.

3 Cooper, S., and Denny-Brown, D., Proc. Roy. Soc., 1927, 102B, 222.

4 Dalma, G., Riv. sper. freniat., 1925, 49, 371.

5 Denny-Brown, D., and Robertson, E. Graeme, Brain, 1933, 56, 149.

6 Denny-Brown, D., Proc. Roy. Soc. Med., 1929-30, 23 (Sect. Neurol.), 3.

7 Ebaugh, F. G., and Stevenson, G. S., Johns Hopkins Hosp. Bull., 1920, 31, 440.

8 ElSBerg, C. A., Surg. Gyncecol. Obst., 1916, 23, 153.

9 Gowers, W. R., Epilepsy and other Convulsive Diseases, 1881.

10 Holmes, Gordon M., Lancet, 1927, i, 957.

11 Howell, W. H., Jour. Exper. Med., 1897, $2,313$.

12 Jackson, J. Hughings, West Riding Asylum Medical Reports, 1876, 6, 266, and Selected Writings, 1931, vol. I, 135.

13 id., Medical Society's Proc., 1886, 10, and Selected Writings, 1931, vol. I, 362.

14 id., Lumleian Lectures, Brit. Med. Jour., 1890, 1, 765, and Selected Writings, 1931, vol. I, 437.

15 Kennedy, F., Arch. Neurol. and Psychiat., 1923, 9, 567.

16 Lennox, W. G., and Coвb, S., Epilepsy, 1928.

17 Leriche, R., Presse méd., 1920, 28, 645.

18 Macdonald, M. E., and CobB, S., this Jou rnal, 1923-4, 4, 228.

19 Martin, J. P., Lancet, 1926, 1, 760.

${ }^{20}$ Mosso, A., Ueber der Kreislauf des Blutes im menschlichen Gehirn, 1881.

${ }^{21}$ Muskens, L. J. J., Epilepsy, 1928.

22 Nawratzki, E., and ARNDt, M., Berlin. klin. Woch., 1899, 36, 662.

${ }^{23}$ Pollock, L. J., and Treadway, W. L., Arch. Int. Med., 1913, 11, 445.

24 Reduich, E., and Pötzl, O., Zeits. f. d. g. Neurol. u. Psychiat., 1910, 3, 492.

25 Robertson, Alexander, Edin. Med. Jour., 1869-70, 15, 513.

${ }^{26}$ Shephard, J. F., Amer. Jour. Psychol., 1906, 17, 522.

27 id., Amer. Jour. Physiol., 1909, 23, xii.

28 Sherrington, C. S., Proc. Roy. Soc., 1909, 81B, 249.

29 Stenvers, H. W., Deut. Zeits. f. Nervenheilk., 1925, 83, 364.

30 Stevenson, L., Christensen, B. E., and Wortis, B., Proc. Assn. Research in Nervous and Mental Disease, vol. VIII, 1929, p. 130.

31 Todd, R. B., Clinical Lectures on Paralysis, 1854.

22 Wilson, S. A. Kinnier, Modern Problems in Neurology, 1928. 